\title{
Fair scheduling in cellular systems in the presence of noncooperative mobiles
}

\author{
Veeraruna Kavitha*+ ${ }^{*}$, Eitan Altman* R. El-Azouzi ${ }^{+}$and Rajesh Sundaresan ${ }^{\times}$ \\ * Maestro group, INRIA, 2004 Route des Lucioles, Sophia Antipolis, France \\ ${ }^{+}$LIA, University of Avignon, 339, chemin des Meinajaries, Avignon, France \\ ${ }^{\times}$IISc, Indian Institute of Science, Bangalore, India 560012
}

\begin{abstract}
We consider the problem of 'fair' scheduling the resources to one of the many mobile stations by a centrally controlled base station (BS). The BS is the only entity taking decisions in this framework based on truthful information from the mobiles on their radio channel. We study the well-known family of parametric $\alpha$-fair scheduling problems from a gametheoretic perspective in which some of the mobiles may be noncooperative. We first show that if the $B S$ is unaware of the noncooperative behavior from the mobiles, the noncooperative mobiles become successful in snatching the resources from the other cooperative mobiles, resulting in unfair allocations. If the BS is aware of the noncooperative mobiles, a new game arises with BS as an additional player. It can then do better by neglecting the signals from the noncooperative mobiles. The BS, however, becomes successful in eliciting the truthful signals from the mobiles only when it uses additional information (signal statistics). This new policy along with the truthful signals from mobiles forms a Nash Equilibrium (NE) which we call a Truth Revealing Equilibrium. Finally, we propose new iterative algorithms to implement fair scheduling policies that robustify the otherwise non-robust (in presence of noncooperation) $\alpha$ fair scheduling algorithms.
\end{abstract}

\section{INTRODUCTION}

Short-term fading arises in a mobile wireless radio communication system in the presence of scatterers, resulting in timevarying channel gains. Various cellular networks have downlink shared data channels that use scheduling mechanisms to exploit the fluctuations of the radio conditions (e.g. 3GPP HSDPA [2] and CDMA/HDR [8] or 1xEV-DO [1]). A central scheduling problem in wireless communications is that of allocating resources to one of many mobile stations that share a common radio channel. Much attention has been given to the design of efficient and fair scheduling schemes that are centrally controlled by a base station (BS) whose decisions depend on the channel conditions of each mobile. These networks use various fairness criteria ([6], [4]) called generalized $\alpha$-fair criteria to design a class of parametric scheduling algorithms (which we henceforth call as $\alpha$-fair scheduling algorithms or $\alpha$-FSA). One special case, proportional fair sharing (PFS), has been intensely analyzed as applied to the CDMA/HDR system. See [12], [8], [7], [20], [3], [11], [17]. These results are applicable to the 3GPP HSDPA system as well. Kushner \& Whiting [15] analyzed the PFS algorithm using stochastic approximation techniques and showed that the asymptotic averaged throughput can be driven to optimize a certain system utility function (sum of logarithms of offset-rates). See also Stolyar [21].
The BS is the only entity taking decisions in all the above methods, and the BS depends crucially on truthful reporting of their channel states by the mobiles. For example, in the frequency-division duplex system, the BS has no direct information on the channel gains, but transmits downlink pilots, and relies on the mobiles' reported values of gains on these pilots for scheduling. A cooperative mobile will truthfully report this information to the BS. A noncooperative mobile will however send a signal that is likely to induce the scheduler to behave in a manner beneficial to the mobile.

In [13], [14] we analyzed efficient scheduling (a special case with $\alpha=0$, wherein the scheduler maximizes the sum throughput at the BS) in presence of noncooperation using a signaling game ([22]). The signaling game can be used only for that special case and an $\alpha$-fair scheduler with $\alpha>0$ cannot be modeled by a signaling game: for $\alpha$-fair schedulers with $\alpha>0$, the utilities of the BS are not expected utilities but are concave combinations of the users' expected utilities. Further, $\alpha$-fair scheduler (with $\alpha>0$ ) has an inherent feedback in its structure (more details in section II) and this feedback makes the study difficult and different from the above paper. This paper has contributions to three main areas:

Networking Aspects: (1) We identify cases where noncooperation results in an unfair bias in the channel assignments in favor of noncooperative mobiles, if the base station is unaware of the noncooperative behavior. (2) We characterize the limitation of the base station, and obtain conditions under which even when it is aware of noncooperation, it is not able to share fairly the resources. (3) We show that the ability to achieve fair sharing, in the presence of noncooperation, depends on the parameter $\alpha$. (4) We design robust iterative algorithms that, under suitable conditions, fairly share the resources even in the presence of noncooperative signaling.

Game theoretical modeling: (1) We model a noncooperative mobile as a rational player that wishes to maximize its throughput. Since the $\alpha$-fair assignment is related to the maximization of a related utility function, one can view the $\mathrm{BS}$ as yet another player. We thus have a game model even if there is a single noncooperative mobile. (2) We formulate three games of which one is a concave game. The formulation of the games turn out to be surprisingly complex. Except for the special case of $\alpha=0$ (where the game can be shown to be equivalent to a matrix game), the games are defined over an infinite set of actions. We are however able to prove the existence and characterize the equilibrium policies 
for two games. (3) The third game arises when the BS is unaware of noncooperation. BS only responds to the mobiles, but in a optimal way. We could model this as a hierarchical game where the mobiles are involved in a game played at the higher level and the BS optimizes some utility at the lower level, unaware of the rationality of the mobiles. (4) To analyze iterative algorithms, we consider a stochastic game with asymptotic time limits of the iterative algorithm as cost criteria.

Design of the networking protocols based on stochastic approximation techniques. (1) We analyze the parametric $\alpha$-fair scheduling algorithm ( $\alpha$-FSA) of [15] in presence of noncooperation. We identified its robustness properties as a function of $\alpha$. (2) Using the knowledge of channel and signal statistics, one can control the excess utilities that the mobiles would have otherwise obtained by noncooperation. This is the basic idea behind robust policies. We then use stochastic approximation based approach to combine estimation (which replaces the knowledge required) and control to design robust fair scheduling algorithms.

We first motivate the problem using a simple example.

\section{A Motivating example}

We consider two users sharing a common channel. User 1 has two channel states with utilities 7 and 3 occurring with probabilities 0.33 and 0.67 respectively. User 2 has constant channel with utility 4 . The BS has to assign the channel to one of the two users for every realization of the channel state and every such assignment rule results in a pair of users's average utilities. The BS uses an $\alpha$-fair scheduler (described in the next section) to fair share these average utilities. First we assume that both users cooperate and report their individual channel states correctly. In figures 1 and 2 (the case with $\delta=0$ ) we plot the average utilities obtained by users under $\alpha$-fair scheduler as a function of the fairness parameter $\alpha$. We make the following observations: (1) For every $\alpha$, the BS always allocates the channel to user 1 if he is in good state. (2) For $\alpha=0$, the expected share of user $1(7 * 0.33)$ is less than that of the user 2 $((1-0.33) * 4)$. This corresponds to efficient scheduling point. (3) For small values of $\alpha$, BS allocates the channel to user 1 only when he is in good state. (4) The expected share of user $\overline{1}$ increases while that of the user 2 decreases as $\alpha$ increases and eventually become equal. To achieve this, the BS starts allocating the channel to the user 1 even when that user is in bad state with increasing probability.

The above scenario depends crucially on the truthful reporting of channel by the user 1 . Now, we consider the scenario when user 1 is noncooperative and tries to increase his utility. He declares to be in good state 7 when actually in bad state 3 with probability $\delta$. BS now observes the user 1 to have good channel with better probability $0.33+\delta * 0.67$ and will schedule as before but based on reported channel conditions. In figures 1,2 we plot the resulting expected utilities of both the users as a function of fairness $\alpha$ for $\delta=0.1, \delta=0.5$ respectively. We observe that the utility of user 1 for small values of $\alpha$ is improved in comparison with its cooperative utility. This also reduces the utility of the user 2 below its cooperative share,

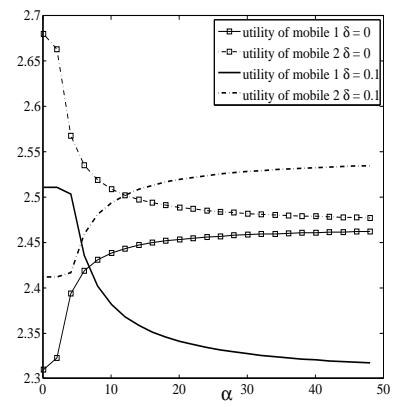

Fig. 1. User utilities versus $\alpha$. for $\delta=0.1$

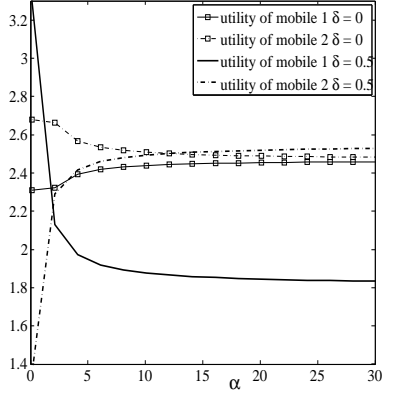

Fig. 2. User utilities versus $\alpha$. for $\delta=0.5$ resulting in unfair allocations. This effect is seen for all values of $\alpha$ less than $\alpha=1.75, \alpha=6.85$ respectively for $\delta=0.5$, $\delta=0.1$. However, for alpha greater than the above values, user 1 loses; in fact its utility gets below its cooperative share, while that of the user 2 is much above the later's cooperative share. The above example indicates the $\alpha$-fair scheduler: (1) might be robust against noncooperation for large values of $\alpha$ (2) fails for smaller values of $\alpha$. (3) the larger the $\delta$ the larger the amount of gain at $\alpha=0$. (4) the larger the $\delta$ the smaller the $\alpha$ till which the mobile gains. As $\alpha$ increases, the two user utilities converge towards equal values at a rate that directly depends upon the difference at $\alpha=0$. This is the reason for the above observation. An important point to note here is that, there is no threshold of $\alpha$ beyond which the scheduler will be robust to all types of noncooperation, i.e., for all values of $\delta$. However one can guess that for max min fairness $(\alpha=\infty)$ the scheduler will be robust. The study of this noncooperation and design of robust policies will be the focus of our paper.

\section{The Problem Setting AND $\alpha$-FAir Scheduler}

The Downlink We consider the downlink of a wireless network with one base station (BS). There are $M$ mobiles competing for the downlink data channel. Time is divided into small intervals or slots. In each slot, one of the $M$ mobiles is allocated the channel. Each mobile $m$ can be in one of the states $h_{m} \in \mathcal{H}_{m}$, where $\mathcal{H}_{m}$ is finite valued. We assume fading characteristics to be independent across the mobiles. Let $\mathbf{h}:=\left[h_{1}, h_{2}, \cdots, h_{M}\right]^{t}$ be the vector of channel gains in a particular slot. The channel gains are distributed according to: $p_{\mathbf{h}}(\mathbf{h})=\prod_{i=1}^{M} p_{h_{i}}\left(h_{i}\right)$, where $\left\{p_{h_{m}} ; m \leq M\right\}$ represent the statistics of the mobile channels. When the mobile's channel state is $h_{m}$, it can achieve a maximum utility given by $f\left(h_{m}\right)$. An example of utility is the rate $f\left(h_{m}\right)=r(m)=\log \left(1+h_{m} \mathrm{SNR}\right)$ where SNR captures the nominal received signal-to-noise ratio under no channel variation.

The decision rule In every slot, the BS has to make scheduling decisions, i.e., allocate the downlink slot to one of the $M$ users, based on the current realization of the channel state vector $\mathbf{h}$. For any set $\mathcal{C}$, let $\mathcal{P}(\mathcal{C})$ be the set of probability measures on $\mathcal{C}$. With that definition, a BS's 
decision is a function $\beta$ that assigns to any given $\mathbf{h}$ an element in $\mathcal{P}(\{1,2, \cdots, M\})$, the probability distribution over the set of users. Thus, $\beta(m \mid \mathbf{h})$ is the probability that the BS schedules current slot to mobile $m$ given channel state vector $\mathbf{h}$.

The $\alpha$-fairness criterion and scheduler We introduce the well known generalized $\alpha$-fair criterion ([4]) where the quantity that we wish to share fairly is the expectation of the random (instantaneous) utilities corresponding to the assignment by the scheduler to the mobiles:

$$
G^{\alpha}(\beta):=\sum_{m=1}^{M} \Gamma^{\alpha}\left(\theta_{m}(\beta)\right)
$$

where $\theta_{m}(\beta):=\mathbb{E}_{\mathbf{h}}\left[f\left(h_{m}\right) \beta(m \mid \mathbf{h})\right]$ is the expected share of mobile $m$ under policy $\beta$ and where the $\alpha$-fair function is

$$
\Gamma^{\alpha}(u):=\left\{\begin{array}{lll}
\log (u), & \text { for } & \alpha=1 \\
\frac{u^{1-\alpha}}{1-\alpha}, & \text { for } & \alpha \neq 1 .
\end{array}\right.
$$

One can view $\beta(. \mid$.$) , the scheduling policy, as a vector in$ $\mathcal{R}^{B}$ space, with $B:=M|\mathcal{H}|$, where $|\mathcal{H}|$ is the cardinality of the product space $\mathcal{H}=\Pi_{m=1}^{M} \mathcal{H}_{m}$. More precisely the domain of optimization is ${ }^{1}$ :

$\mathcal{D}:=\left\{\beta(. \mid):. \sum_{m=1}^{M} \beta(m \mid \mathbf{h})=1, \beta(m \mid \mathbf{h}) \geq 0\right.$ for all $\left.\mathbf{h}, m\right\}$.

The objective function $G^{\alpha}$ given by (1), is concave and continuous in $\beta$ for each $\alpha$, while the domain $\mathcal{D}$ is compact and convex. Hence there always exists a cooperative $\alpha$-fair scheduling BS strategy $\beta^{*}$ :

$$
\beta^{*}(. \mid .) \in \arg \max _{\beta \in \mathcal{D}} G^{\alpha}(\beta) .
$$

Remarks II-1: We may view the BS's schedule as a static optimization problem that corresponds to a single choice of $\beta$. Notice that the optimal schedule $\beta^{*}$ maximizes some function of the expected shares of utilities. This expected share depends on assignments at all channel states, and is therefore a joint optimization problem. This feature arises when $\alpha>0$. When $\alpha=0$ the problem is separable, and the solution $\beta^{*}(\cdot \mid \mathbf{h})$ for a given $\mathbf{h}$ depends only on that $\mathbf{h}$. Indeed, for $\alpha>0$, the implicit equation (3) below highlights a certain 'feedback' that is absent in case with $\alpha=0$. This makes the present study significantly different from our previous work on efficient scheduling with strategic mobiles ([13]).

Below we show a key (feedback) property of $\alpha$ fair schedulers. Define $\beta^{*}$ as the vector (fixed point) that satisfies (if it exists) the following:

$$
\beta^{*}(m \mid \mathbf{h})=\frac{1_{\left\{m \in \arg \max _{j} d \Gamma^{\alpha}\left(\theta_{j}\left(\beta^{*}\right)\right) f\left(h_{j}\right)\right\}}}{\left|\arg \max _{j} d \Gamma^{\alpha}\left(\theta_{j}\left(\beta^{*}\right)\right) f\left(h_{j}\right)\right|}
$$

where $d \Gamma^{\alpha}\left(\theta_{j}(\beta)\right):=\left.\frac{d \Gamma^{\alpha}}{d u}\right|_{u=\theta_{j}(\beta)}$ is the derivative of $\Gamma^{\alpha}$ with respect to (w.r.t.) $u$, evaluated at $\theta_{j}(\beta)$. We now have

Lemma 1: If there is a $\beta^{*}$ satisfying (3), then $\beta^{*}$ is a global maximizer of the objective function in (2) over domain $\mathcal{D}$ and hence is an $\alpha$-fair scheduler.

\footnotetext{
${ }^{1}$ For a given channel realization $\mathbf{h}$ the BS will choose the mobile randomly according to the measure $\beta(. \mid \mathbf{h})$.
}

Let $\Theta:=\left[\begin{array}{lll}\theta_{1} & \cdots & \theta_{M}\end{array}\right]^{T}, \Theta(\beta):=\left[\begin{array}{lll}\theta_{1}(\beta) & \cdots & \theta_{M}(\beta)\end{array}\right]^{T}$ and $\Theta(\mathcal{D}):=\{\Theta(\beta): \beta \in \mathcal{D}\}$. The map $\Theta \mapsto \sum_{m} \Gamma^{\alpha}\left(\theta_{m}\right)$ is strictly concave. Hence, there exists an unique maximizer (of the expected assigned shares) over the convex set $\Theta(\mathcal{D})$ :

$$
\Theta^{*}=\max _{\Theta \in \Theta(\mathcal{D})} \sum_{m} \Gamma^{\alpha}\left(\theta_{m}\right) \text {. }
$$

Hence, if there is a $\beta^{*}$ satisfying (3) then $\Theta^{*}=\Theta\left(\beta^{*}\right)$. Further, any $\beta^{*}$ which is a global maximum of the objective function (2) satisfies the 'efficiency' property: whenever $f\left(h_{m}\right)>f\left(h_{m}^{\prime}\right)$

$$
\begin{aligned}
\text { either } & \beta^{*}\left(m \mid h_{m}, \mathbf{h}_{-m}\right)>\beta^{*}\left(m \mid h_{m}^{\prime}, \mathbf{h}_{-m}\right) \\
\text { or } & \beta^{*}\left(m \mid h_{m}, \mathbf{h}_{-m}\right)=\beta^{*}\left(m \mid h_{m}^{\prime}, \mathbf{h}_{-m}\right) \in\{0,1\}
\end{aligned}
$$

for all $\mathbf{h}_{-m} \in \Pi_{j \neq m} \mathcal{H}_{j}$ and for all $m$.

Proof : Please refer to Appendix B.

The above Lemma 1 gives the exact characterization of an optimal solution of $\alpha$-fairness problem (3). It further talks about the efficiency of every possible $\alpha$-fair solution (5): the assignment for particular state $\left(h_{m}\right)$ for any mobile $m$ increases with the increase in the utility $\left(f\left(h_{m}\right)\right)$ of the state. This property is used in the analysis under noncooperation. A part of Lemma 1, regarding the possible solution (3), when restricted to proportional fairness, is already stated in [16].

Remarks II-2: The solution (3) explicitly shows the feedback we mentioned in Remark II-1. This solution has already been used in practical scenarios ([16]) to achieve 'fair' scheduling: The $\alpha$-fair solution for the dynamic setting with ergodic channel states is the optimal $\beta$ that fair shares the time average utilities over a single realization of a whole sample path ${ }^{2}$. In fact, the solution (3) under ergodicity can be implemented by the following procedure: 1) At any time slot $k$, obtain the scheduling decision using the current channel vector $\mathbf{h}_{k}$ and using the time averaged assigned utilities obtained till the last step $\left\{\theta_{m, k-1}\right\}$ in place of $\left\{\theta_{m}\left(\beta^{*}\right)\right\}$ of $\left.(3) ; 2\right)$ Update (in the obvious way) the time averaged assigned utilities up to step $k\left\{\theta_{m, k}\right\}$ using the current scheduling decision.

\section{PROBLEM FORMULATION UNDER NON COOPERATION}

In every slot, the BS needs the knowledge of $\mathbf{h}$ for scheduling purposes. In practice, mobile $m$ estimates channel $h_{m}$ using the pilot signals sent by BS. We assume perfect channel estimation. The mobiles send signals $\left\{s_{m}\right\}$ to $\mathrm{BS}$, as indications of the channel gains. Thus BS does not have direct access to channel state $\mathbf{h}$, but instead has to rely on the mobiles for it. If the mobiles have selfish motives, they can signal a better channel condition to grab the channel even when their channel is bad.

The main purpose of this paper is to study the effect of this noncooperation on the $\alpha$-fair scheduler (2). We assume that

${ }^{2}$ For ergodic channels under appropriate conditions on the function $g$,

$$
\lim _{K \rightarrow \infty} \frac{1}{K} \sum_{k=1}^{K} g\left(\mathbf{h}_{k}\right)=\mathbb{E}_{\mathbf{h}}[g(\mathbf{h})]
$$

We are interested in a particular function $g(\mathbf{h})=f\left(h_{m}\right) \beta(m \mid \mathbf{h})$ whose average is exactly $\theta_{m}(\beta)$. 
signals are chosen from the channel space itself, i.e., $s_{m} \in \mathcal{H}_{m}$ for all mobiles. We shall consider two types of scenarios :

Hierarchical game G1: The BS is unaware of the possible noncooperative behavior from the mobiles and applies the $\alpha$ fair scheduler (2) to the signals $\mathbf{s}=\left[s_{1}, \cdots, s_{M}\right]^{t}$ (as if they were the true channel values). The mobiles are aware of BS's scheduler, signal to optimize their own goals. When the base station is unaware, we model this game as hierarchical game with two levels: where leaders, the noncooperative mobiles, involve in a game problem while BS, the follower does the optimization. In this game, there is no common knowledge: the base station does not know the rationality of the mobiles. This game is related to that discussed by Aumann in [5] through many examples. For several years it has been thought that the assumption of common knowledge of rationality for the players in the game was fundamental. It turns out that, in $\mathrm{N}$ player games, common knowledge of rationality is not needed as an epistemic condition for equilibrium strategies (see [5]).

A game approach: The BS is modeled as an additional player in a one-shot game. When the BS becomes aware of the possible noncooperation, it could implement better policies to do better. We first consider a $M_{1}+1$ player game G2, where the BS schedules still only using the signals from the mobiles. Because of its awareness, it could do better than the situation of game G1, but however will not be successful in compelling the mobiles to reveal the truthful signals (Section V-A). In Section V-B we construct more intelligent (which require more information) BS policies which would be robust against noncooperation: the new robust BS policies and the truthful signals from the mobiles form a Nash Equilibrium. This we refer as game G3.

We now introduce the important concepts and definitions that are used in the paper. These are more specific to the first two game scenarios. The corresponding definitions and concepts may vary slightly for the game G3 and the differences are explained directly in Section V-B.

Common Knowledge : Channel statistics $\left\{p_{h_{m}} ; m \leq M\right\}$ of all mobiles is a common knowledge, i.e., known to all the mobiles and the BS. We also assume that, the information about which mobiles are noncooperative, is a common knowledge in case of the last two games G2 and G3. If the rational BS does not know which mobiles are cooperative, it will treat every mobile as noncooperative.

Mobile Policies : Some mobiles (with indices $1 \leq m \leq M_{1}$ where $\left(0 \leq M_{1} \leq M\right)$ are assumed to be noncooperative. A policy of mobile $m$ is a function $\left\{\mu_{m}\left(. \mid h_{m}\right)\right\}$ that maps a state $h_{m}$ to an element in $\mathcal{P}\left(\mathcal{H}_{m}\right)$.

BS Policies : A policy of the BS is a function which maps every signal vector $\mathbf{s}$ to a scheduler $\beta \in \mathcal{P}(\{1,2, \cdots, M\})$. These policies are used in major part of the paper, while more complicated policies are considered in section V-B.

Utilities for a given set of strategies : The instantaneous/sample utility of the mobile $m$ depends only upon the true channel $h_{m}$ and the BS decision $\beta$ and is given by :

$$
U_{m}\left(s_{m}, h_{m}, \beta\right)=1_{\{\beta=m\}} \min \left\{f\left(h_{m}\right), f\left(s_{m}\right)\right\}^{3} .
$$

Define the following to exclude mobile $m$ :

$$
\begin{array}{rlc}
\mathbf{h}_{-m} & := & {\left[h_{1}, \cdots, h_{m-1}, h_{m+1}, \cdots, h_{M}\right],} \\
p_{\mathbf{h}_{-m}}\left(\mathbf{h}_{-m}\right) & :=\Pi_{j \neq m} p_{h_{j}}\left(h_{j}\right), \\
\mu_{-m}\left(\mathbf{s}_{-m} \mid \mathbf{h}_{-m}\right) & := & \Pi_{j \neq m ; j \leq M_{1}} \mu_{j}\left(s_{j} \mid h_{j}\right) \\
& \quad \Pi_{j \neq m ; j>M_{1}} \delta\left(h_{j}=s_{j}\right) .
\end{array}
$$

Also define, $\mu=\left\{\mu_{m} ; m \leq M_{1}\right\}$ to represent strategy profile:

$$
\mu(\mathbf{s} \mid \mathbf{h}):=\Pi_{1 \leq j \leq M_{1}} \mu_{j}\left(s_{j} \mid h_{j}\right) \Pi_{j>M_{1}} \delta\left(h_{j}=s_{j}\right) .
$$

With the above definitions, each noncooperative user chooses its strategy $\mu_{m}$ in such a way as to maximize its own utility:

$$
U_{m}^{\alpha}(\mu, \beta)=\mathbb{E}_{\mathbf{h}}\left[\sum_{\mathbf{s}} U_{m}\left(s_{m}, h_{m}, m\right) \beta(m \mid \mathbf{s}) \mu(\mathbf{s} \mid \mathbf{h})\right]
$$

Under the $\alpha$-fair criterion (1), the natural selection of utility for BS will be:

$$
U_{B S}^{\alpha}(\mu, \beta)=\sum_{m} \Gamma^{\alpha}\left(U_{m}^{\alpha}(\mu, \beta)\right) .
$$

Throughout when $\arg \max S$ has more than one element, by $i=\arg \max S$ we mean $i \in \arg \max S$. By $j:=\arg \max S$ we mean that $j$ is a chosen element of $\arg \max S$.

ASA, ATA Utilities : When mobile signals do not match the true channel values, the game under consideration will have two important average utilities for any given strategy profile $(\mu, \beta)$ : (1) average signaled utilities under assignment $\beta$ (ASA) utility, which a (more intelligent) BS can observe, and (2) average true and assigned (ATA) utility, which is the true average utility gained by the mobile and whose value cannot be estimated (as long as the mobile is noncooperative) at the BS. These are defined by

$$
\begin{aligned}
& U_{m}^{A S A}(\mu, \beta):=\mathbb{E}_{\mathbf{h}}\left[\sum_{\mathbf{s}} f\left(s_{m}\right) \mu(\mathbf{s} \mid \mathbf{h}) \beta(m \mid \mathbf{s})\right] \\
& U_{m}^{A T A}(\mu, \beta) \\
& \quad:=\mathbb{E}_{\mathbf{h}}\left[\sum_{\mathbf{s}} \min \left\{f\left(h_{m}\right), f\left(s_{m}\right)\right\} \mu(\mathbf{s} \mid \mathbf{h}) \beta(m \mid \mathbf{s})\right] .
\end{aligned}
$$

Indeed, it is easy to observe that the utility of mobile $m$ is its ATA utility, i.e., $U_{m}^{\alpha}(\mu, \beta)=U_{m}^{A T A}(\mu, \beta)$.

Truth Revealing Strategy : In the following, by truth revealing strategy at mobile $m$ we mean the strategy

$$
\mu_{m}^{T}\left(s_{m} \mid h_{m}\right)=1_{\left\{s_{m}=h_{m}\right\}} \text { for all } h_{m}, s_{m} \in \mathcal{H}_{m},
$$

which signals the true channel state. Let $\mu^{T}:=\left(\mu_{1}^{T}, \cdots, \mu_{M}^{T}\right)$. Under truthful strategies $\mu^{T}$, ATA and ASA utilities coincide. For any BS policy $\beta$, if strategy profile $\left(\mu^{T}, \beta\right)$ forms a Nash Equilibrium, then we call the NE as a Truth Revealing Equilibrium (TRE).

\footnotetext{
${ }^{3}$ The mobile achieves rate $f\left(h_{m}\right)$ even if the BS allocates it a higher rate $f\left(s_{m}\right)$ because of the inflated signal $s_{m}$ sent by the noncooperative mobile. The justification of this is provided in detail in Appendix C (we used similar assumption also in [13], [14]).
} 
Cooperative Shares : Best response of BS to truthful signals $\mu^{T}$ is any maximizer $\beta^{*}$ of $G^{\alpha}(1)$. By Lemma 1 , the best response results in unique maximum average ATA utilities,

$$
\theta_{m}^{\alpha c}:=\theta_{m}\left(\beta^{*}\right)=U_{m}^{\alpha}\left(\mu^{T}, \beta^{*}\right),
$$

which will be referred as Cooperative Shares.

Contrast between hierarchical optimization and the game perspective: Recall that computing a fair assignment by BS involves maximization of (1). Thus in the first scenario, when mobiles choose profile $\mu$, the unaware BS fair shares ASA utilities under $\mu$ by maximizing (12) (given in the next section). However, what needs fair sharing is the ATA utilities. This is achieved via the game perspective, wherein the rational BS tries to fair share the ATA utilities gained by the mobiles.

We study the various scenarios via three games mentioned above.

\section{SCHEDULING UNDER NONCOOPERATION : HIERARCHICAL GAME PROBLEM G1}

We consider the scenario in which the BS is unaware of the presence of noncooperative mobiles. As in cooperative setting, the BS schedules (using optimal scheduler (2)) the channel to one of the mobiles using the mobile signals, assuming them to reflect the channel state perfectly. The mobiles, aware of BS's scheduler, maximize their utilities.

Utilities of G1: For any given mobile strategy profile $\mu$, let the induced signal probabilities be represented by $p_{\mathbf{s}}$, i.e., $p_{\mathbf{s}}(\mathbf{s})=\sum_{\mathbf{h}} p_{\mathbf{h}}(\mathbf{h}) \mu(\mathbf{s} \mid \mathbf{h})$. Since the BS observes $p_{\mathbf{s}}$ (instead of $p_{\mathbf{h}}$ ), it assumes the expected shares of mobile $m$ to be $\theta_{m}(\mu, \beta):=\mathbb{E}_{p_{\mathbf{s}}}\left[f\left(s_{m}\right) \beta(m \mid \mathbf{s})\right]$ and hence maximizes,

$$
\left.U_{B S}^{A S A}(\beta, \mu)=\sum_{m} \Gamma^{\alpha}\left(\theta_{m}(\mu, \beta)\right)\right) .
$$

One can identify that $\theta_{m}(\mu, \beta)$ are the ASA utilities. The equilibrium appropriate to this scenario is Stackelberg Equilibrium. Stackelberg Equilibrium for G1: is a profile $\left(\beta_{\mu}^{*}, \mu^{*}\right)$ which satisfies the following for all $m$ :

$$
\begin{aligned}
\beta_{\mu}^{*} & =\arg \max _{\beta} U_{B S}^{A S A}(\beta, \mu), \\
\mu_{m}^{*} & =\arg \max _{\mu_{m}} U_{m}^{A T A}\left(\left(\mu_{m}, \mu_{-m}^{*}\right), \beta_{\left(\mu_{m}, \mu_{-m}^{*}\right)}^{*}\right) .
\end{aligned}
$$

We now present some examples in which a user $m$ deviates unilaterally from $\mu^{T}$ and increases its utility above its cooperative share, resulting in unfair allocations. These examples do not have TRE for G1, i.e., truthful strategy profile $\mu^{T}$ is not a part of any Stackelberg Equilibrium of G1. In particular for (12), we consider $\alpha$-fair scheduler given by (3). This scheduler is a widely used practical solution (see Remark II-2), $\alpha$-FSA being one of them.

\section{A. Asymmetric Examples}

1) Proportional fair scheduler $(\alpha=1)$ : We continue with the motivating example given in Section I. User 1 has a single state with utility $a$. User 2 has 2 states with respective utilities given by $r b, b$ and with $r>1$. The respective probabilities to be in one of these states are $p,(1-p)$ with $p \in(1 /(1+r), 1 / 2)$.
Using (3), one can easily estimate $\beta^{*},\left\{\theta_{m}\left(\beta^{*}\right)\right\}$ to be:

$$
\begin{array}{ll}
\beta^{*}(2 \mid a, r b) & =1,
\end{array} \quad \begin{aligned}
\beta^{*}(1 \mid a, b) & =1, \\
\theta_{1}\left(\beta^{*}\right) & =a(1-p)
\end{aligned} \quad \text { and } \quad \theta_{2}\left(\beta^{*}\right)=r b p .
$$

Note that $\theta_{1}\left(\beta^{*}\right), \theta_{2}\left(\beta^{*}\right)$ are the mobile's cooperative shares. It is important to note here that $\beta^{*}$ satisfying (3) exist only if $p \in(1 /(1+r), 1 / 2)$ as in this case :

$$
\begin{aligned}
d \Gamma^{\alpha}\left(\theta_{2}\left(\beta^{*}\right)\right) r b & =\frac{r b}{r b p}>\frac{a}{a(1-p)}=d \Gamma^{\alpha}\left(\theta_{1}\left(\beta^{*}\right)\right) a \\
d \Gamma^{\alpha}\left(\theta_{2}\left(\beta^{*}\right)\right) b & =\frac{b}{r b p}<\frac{a}{a(1-p)}=d \Gamma^{\alpha}\left(\theta_{1}\left(\beta^{*}\right)\right) a .
\end{aligned}
$$

Suppose user 2 signals $r b$ (when actually in state $b$ ) with probability $q$, i.e., $\mu_{2}(r b \mid b)=q$. Then user's maximum ASA rates (note that $\beta_{q}^{*}=\beta^{*}$ defined in (14)) are:

$$
U_{1}^{A S A}\left(q, \beta_{q}^{*}\right)=(1-p-q) a, \quad U_{2}^{A T A}\left(q, \beta_{q}^{*}\right)=r b(p+q)
$$

respectively whenever

$$
\frac{r b}{(p+q) r b}>\frac{a}{a(1-p-q)}>\frac{b}{r b(p+q)} .
$$

With this, the mobile 2 obtains an improved ATA utility $U_{2}^{A T A}\left(q, \beta_{q}^{*}\right)=r b p+b q>\theta_{2}\left(\beta^{*}\right)$, i.e., mobile 2 is successful in improving its utility (above it's cooperative share) by signaling noncooperatively. The maximum possible value of $q$ is $q=(0.5-p)$.

2) Extension to general $\alpha$ : One can extend the above to general $\alpha$, an $\alpha$-fair scheduler satisfying (3) exists if,

$$
(r b)^{\alpha-1} p^{\alpha}<a^{\alpha-1}(1-p)^{\alpha}<r(r b)^{\alpha-1} p^{\alpha} .
$$

From above, as $\alpha$ increases, $p$ for which (3) exists reduces and thus given $(a, r, b, p)$, there exists a maximum $\alpha_{\max }$, beyond which there does not exist $\alpha$-fair scheduler of the type (3). However another type of alpha-fair scheduler exist; for example for max-min fairness (when $\alpha=\infty, \theta_{1}^{*}=\theta_{2}^{*}$ ) a $\alpha$-fair scheduler $\left\{\beta^{*}(1 \mid r b, a), \quad \beta^{*}(1 \mid b, a)\right\}$ given by:

$$
\begin{gathered}
\beta^{*}(1 \mid r b, a)=\frac{a}{r b p+a p} ; \beta^{*}(1 \mid b, a)=0 \text { if } a(1-p)<r b p \\
\beta^{*}(1 \mid b, a)=\frac{a(1-p)-r b p}{(b+a)(1-p)} ; \beta^{*}(1 \mid r b, a)=0 \quad \text { else. }
\end{gathered}
$$

When $\alpha$-fair scheduler (3) exists the noncooperative mobile benefit; the maximum $q(\alpha)$ satisfies:

$$
(p+q(\alpha))^{\alpha}(r b)^{\alpha-1}=a^{\alpha-1}(1-p-q(\alpha))^{\alpha} .
$$

For example with $a=4, r=3, b=3, p=0.33$ the maximum $\alpha$ for which $\alpha$-fair scheduler (3) exists is 7.9 and user 1 can benefit by signaling with $q=.05$ for all $\alpha \leq 4$.

3) Generalization to more states and general $\alpha$ : Consider two asymmetric users under the following assumptions :

N.1 The cooperative $\alpha$-fair solution $\beta^{*}$ (3) exists and without loss of generality let $1=\arg \max _{m} \theta_{m}^{\alpha c}$.

N.2 There exists an $i>1$ such that,

$$
\eta:=\inf _{h_{2} \in \mathcal{H}_{2}} d \Gamma^{\alpha}\left(\theta_{1}^{\alpha c}\right) f\left(h_{1, i-1}\right)-d \Gamma^{\alpha}\left(\theta_{2}^{\alpha c}\right) f\left(h_{2}\right)>0,
$$

where $\mathcal{H}_{1}=\left\{h_{1,1}, \cdots, h_{1, N_{1}}\right\}$ are arranged such that $f\left(h_{1,1}\right)>f\left(h_{1,2}\right)>\cdots>f\left(h_{1, N_{1}}\right)$. 
Lemma 2: Under assumptions N.1-N.2, there exist a non truth revealing policy $\mu_{1}^{\delta}$ for mobile 1 such that its ATA utility $U_{1}^{A T A}\left(\mu_{1}^{\delta},\left(f, \beta_{\mu_{1}^{\delta}}^{*}\right)\right)$ is larger than its cooperative share $\theta_{1}^{\alpha c}$.

Proof : The proof is available in Appendix B.

\section{B. Symmetric Case}

We consider a simple symmetric two mobile example. The mobiles have two states with utilities $a_{1}, a_{2}$ occurring respectively with probabilities $p_{1}, p_{2}$. Let $a_{1}=r a_{2}, p_{1}=p p_{2}$ with $r>1, p>0$. Under truthful signaling, by Lemma 1 , an $\alpha$-fair optimal BS policy (for any $\alpha$ ) is given by:

$$
\begin{array}{r}
\beta^{*}\left(1 \mid a_{1}, a_{1}\right)=1 / 2=\beta^{*}\left(1 \mid a_{2}, a_{2}\right), \quad \beta^{*}\left(1 \mid a_{1}, a_{2}\right)=1, \\
\beta^{*}\left(1 \mid a_{2}, a_{1}\right)=0
\end{array}
$$

with equal cooperative shares

$$
\begin{aligned}
\theta_{1}\left(\beta^{*}\right)=\theta_{2}\left(\beta^{*}\right) & =\left(\frac{p_{1}^{2}}{2}+p_{1} p_{2}\right) a_{1}+p_{2}^{2} \frac{a_{2}}{2} \\
& =p_{2}^{2} a_{2}\left(\frac{p^{2} r+1}{2}+p r\right) .
\end{aligned}
$$

Without loss of generality say mobile 1 deviates unilaterally from his truthful strategy with $\mu_{1}\left(a_{1} \mid a_{2}\right)=t$. If mobile 1 was successful, his reported rate would be greater than $\theta_{1}\left(\beta^{*}\right)$ and this rate he would have obtained only when his declared state is $a_{1}$ with mobile 2's being $a_{2}$. Thus, mobile 1 will be successful with maximum ASA utilities $(\alpha=1)$ :

$$
\begin{aligned}
U_{1}^{A S A} & =\left(p_{1} a_{1}+p_{2} t a_{1}\right) p_{2}=(p+t) p_{2}^{2} a_{1} \text { and } \\
U_{2}^{A S A} & =1 p_{1} a_{1}+p_{2}(1-t) p_{2} a_{2}=\left(p r+(1-t) p_{2}\right) p_{2} a_{2}
\end{aligned}
$$

and the corresponding ATA utility,

$$
U_{1}^{A T A}=\left(p_{1} a_{1}+p_{2} t a_{2}\right) p_{2}=(p r+t) p_{2}^{2} a_{2}
$$

if the following conditions are met:

$$
\frac{a_{1}}{U_{1}^{A S A}}>\frac{a_{2}}{U_{2}^{A S A}} \text { and } \theta_{1}\left(\beta^{*}\right)<U_{1}^{A T A},
$$

i.e., if $t$ satisfies:

$$
\frac{1}{(p+t) p_{2}}>\frac{1}{\left(p r+(1-t) p_{2}\right)} \text { and } \frac{p^{2} r+1}{2}<t .
$$

\section{Robustness at large $\alpha$}

For small values of $\alpha, \alpha$ fair scheduler fails. However we see a different phenomenon at higher $\alpha$. As $\alpha$ increases to infinity, the 'fairness' increases and the expected shares, i.e., ATA utilities, of all the mobiles tend to becoming equal ([18]), provided all the mobiles signal truthfully. However, in presence of noncooperation, it will be the ASA utilities that start becoming equal for higher values of $\alpha$. This results in all the cooperative (ATA equal ASA utilities) mobiles getting equal ATA shares which will be bigger than that for the noncooperative (ATA are strictly less than ASA utilities) mobiles. Thus the $\alpha$-fair scheduler (2) itself becomes more and more robust towards noncooperation as fairness factor $\alpha$ increases, in spite of the $B S$ 's unawareness of the noncooperation. ${ }^{4}$ This effect is seen

\footnotetext{
${ }^{4}$ However as noticed in motivating example, we can only say the max min fairness will be robust against all types of noncooperation from mobiles and cannot identify an $\alpha$ beyond which the scheduler will be robust.
}

in the motivating example as well as in Figure 3 given in a later section. In Figure 3, the noncooperative mobile's ATA utility diminishes as $\alpha$ increases and goes below its cooperative share beyond $\alpha=1.2$ and further, the cooperative mobile gets more share than its cooperative share for these large values of $\alpha$.

\section{Scheduling Under Noncooperation : Game THEORETIC STUDY}

In this section the BS knows about noncooperative behavior of mobiles and is considered as an additional player resulting in the $M_{1}+1$ player game.

\section{A. BS Scheduling policies of section IV : Game G2}

In contrast to section IV, the BS knows the mobiles that are noncooperative. The resulting game is a one-shot concave game: the utility of mobile $m(6)$ is linear in its policy $\mu_{m}$ while that of the BS (7) is continuous and concave in its policy $\beta$. By [19], this game always has a $\mathrm{NE}^{5}\left(\mu^{*}, \beta^{*}\right)$ which satisfies, for all $m$,

$$
\begin{aligned}
\mu_{m}^{*} & =\arg \max _{\mu_{m}} U_{m}^{\alpha}\left(\left(\mu_{m}, \mu_{-m}^{*}\right), \beta^{*}\right) \\
\text { and } \beta^{*} & =\arg \max _{\beta} U_{B S}^{\alpha}\left(\mu^{*}, \beta\right) .
\end{aligned}
$$

For game G2 we obtain a 'babbling' equilibrium. We further show that G2 does not have a TRE.

1) G2 has Babbling NE: We will now show that this game has a Nash equilibrium where the BS neglects the signals from the noncooperative users. Let $\mathbf{h}^{>M_{1}}:=\left[h_{M_{1}+1}, \cdots h_{M}\right]^{t}$ represent the channel states of the cooperative mobiles. With $\theta_{m}^{>M_{1}}(\beta):=E_{\mathbf{h}}\left[f\left(h_{m}\right) \beta\left(m \mid \mathbf{h}^{>M_{1}}\right)\right]$, the BS maximizes:

$$
\sum_{m} \Gamma^{\alpha}\left(\theta_{m}^{>M_{1}}(\beta)\right) .
$$

We note here that for any non cooperative mobile,

$$
\theta_{m}^{>M_{1}}(\beta)=E\left[f\left(h_{m}\right)\right] E_{\mathbf{h}>M_{1}}\left[\beta\left(m \mid \mathbf{h}^{>M_{1}}\right)\right] \text { for } m \leq M_{1} .
$$

As in Lemma 1 , there always exists a $\beta$ maximizing (15). Call one such $\beta$ by $\beta^{>M_{1} *}$. Choose any mobile profile $\mu$ which satisfies for all $m \leq M_{1}, \mu_{m}\left(s_{m} \mid h_{m}\right)=0$ for all $h_{m}, s_{m}$ with $f\left(s_{m}\right)<f\left(h_{m}\right)$. It is easy to see that $\left(\mu, \beta^{>M_{1} *}\right)$ forms a Nash Equilibrium. Note here that a noncooperative mobile $m$ can obtain the utility $\theta_{m}^{>M_{1}}\left(\beta^{>M_{1} *}\right)$ only if it signals better than its channel true value (as only in this case $\min \left\{f\left(h_{m}\right), f\left(s_{m}\right)\right\}=f\left(h_{m}\right)$ ) and hence the requirement of above condition on the set of mobile strategies.

This is a NE at which the BS ignores the signals from the noncooperative mobiles and is similar in sense to the Babbling equilibrium defined in the context of signaling games ([22]). Hence we chose to call this also as Babbling equilibrium.

We end this subsection with a useful, practically implementable $\alpha$-fair scheduler involving only cooperative signals

\footnotetext{
${ }^{5}$ Note that when adding further concave constraints the game remains concave even if the constraints are coupled [19]. We thus obtain equilibrium also for constrained versions of the game. Examples of such constraints are: the (possible weighted) sum of throughputs is bounded by a constant.
} 
(if exists). Define (if exists),

$$
\begin{aligned}
\beta^{>M_{1} *}\left(m \mid \mathbf{h}^{>M_{1}}\right) & =\frac{1_{\left\{m \in \mathcal{A}^{>M_{1}}\left(\mathbf{h}^{>M_{1}, \beta^{>} M_{1} *}\right)\right\}}}{\left|\mathcal{A}^{>M_{1}}\left(\mathbf{h}^{>M_{1}}, \beta^{>M_{1} *}\right)\right|} \\
\mathcal{A}^{>M_{1}}\left(\mathbf{h}^{>M_{1}}, \beta\right) & =\arg \max _{j} d \Gamma^{\alpha}\left(\theta_{j}^{>M_{1}}(\beta)\right) u_{j} \\
\text { with } u_{j} & =f\left(h_{j}\right) 1_{\left\{j>M_{1}\right\}}+\mathbb{E}\left[f\left(h_{j}\right)\right] 1_{\left\{j \leq M_{1}\right\}} .
\end{aligned}
$$

Using similar steps which obtained (3), one can show that $\beta^{>M_{1} *}$ is a maximizer of (15).

2) G2 has No TRE: We now examine the existence of the desired TRE. The case of $\alpha=0$, the efficient scheduling is studied in [13]. In [13], G2 corresponding to efficient scheduling was modeled by a signaling game and it is shown that the game G2 has only babbling equilibrium as NE and hence does not have a TRE. We will now consider the case $\alpha>0$. If the $M_{1}+1$ player game were to have a TRE, the corresponding (equilibrium) strategy of the BS, by definition the NE, should be the best response to mobile's truthful strategies $\mu^{T}$ and hence will be maximizer of $U_{B S}^{\alpha}\left(\mu^{T}, \beta\right)=G^{\alpha}(\beta)$. Hence, the best response for truth revealing strategy profile $\mu^{T}$ indeed equals one of the maximizers of Lemma 1, which satisfies the efficiency property (5).

Let $\bar{\beta}^{*}$ be any maximizer of Lemma 1 . The strategy profile $\left(\mu^{T}, \bar{\beta}^{*}\right)$ does not form a NE because: Let $\tilde{m}$ be any mobile with non zero cooperative share and let $\tilde{h}$ be its channel value with largest utility, i.e., let $\tilde{h}=\arg \max _{h \in \mathcal{H}_{\tilde{m}}} f(h)$. The mobile by changing its policy from truthful signals $\mu_{\tilde{m}}^{T}$ to $\mu_{\tilde{m}}\left(s_{\tilde{m}} \mid h_{\tilde{m}}\right):=1_{\left\{s_{\tilde{m}}=\tilde{h}\right\}}$ for all $h_{\tilde{m}}, s_{\tilde{m}}$ increases its ATA utilities as by (5) for any $\mathbf{h}_{-\tilde{m}}$ and for any $h \neq \tilde{h} \in \mathcal{H}_{\tilde{m}}$, $\bar{\beta}^{*}\left(\tilde{m} \mid \tilde{h}, \mathbf{h}_{-\tilde{m}}\right) \geq \bar{\beta}^{*}\left(\tilde{m} \mid h, \mathbf{h}_{-\tilde{m}}\right)$, and hence,

$$
\begin{aligned}
& \left.U_{\tilde{m}}^{A T A}\left(\left(\mu_{-\tilde{m}}^{T} \mu_{\tilde{m}}\right), \bar{\beta}^{*}\right)-U_{\tilde{m}}^{A T A}\left(\mu^{T}, \bar{\beta}^{*}\right)\right) \\
& =\sum_{\mathbf{h}} p_{\mathbf{h}}(\mathbf{h})\left(\bar{\beta}^{*}\left(\tilde{m} \mid \tilde{h}, \mathbf{h}_{-\tilde{m}}\right) f\left(h_{m}\right)-\bar{\beta}^{*}(\tilde{m} \mid \mathbf{h}) f\left(h_{m}\right)\right) \\
& =\sum_{\mathbf{h}} p_{\mathbf{h}}(\mathbf{h}) f\left(h_{m}\right)\left(\bar{\beta}^{*}\left(\tilde{m} \mid \tilde{h}, \mathbf{h}_{-\tilde{m}}\right)-\bar{\beta}^{*}(\tilde{m} \mid \mathbf{h})\right)>0 .
\end{aligned}
$$

Strict greater than zero results in the last line for all $\alpha>$ 0 , as all the mobiles obtain non zero utility under an alpha fair scheduler. Thus, the mobile $\tilde{m}$ can improve its utility by unilaterally moving away from $\mu_{m}^{T}$, contradicting the definition of NE.

Thus the BS, even when aware of the noncooperation, is not successful in eliciting the truthful signals. In the following we construct more intelligent policies which induce a TRE. Hence, $B S$ has to use more intelligent scheduling algorithms to be robust against noncooperation.

\section{B. Robust BS Policies : Game G3 has TRE}

BS can estimate statistics $p_{\mathbf{s}}$ after sufficient observation of the mobile signals. We use $p_{\mathbf{s}}$ to build robust policies for BS which give us the desired TRE. The policy of BS now maps every ordered pair of signal and signal statistics $\left(\mathbf{s}, p_{\mathbf{s}}\right)$ to an ordered pair $(\Phi, \beta)=\left\{\left(\phi_{m}\left(\mathbf{s}, p_{\mathbf{s}}\right), \beta\left(. \mid \mathbf{s}, p_{\mathbf{s}}\right)\right)\right\}$ with allocation $\phi_{m}(\mathbf{s}) \leq f\left(s_{m}\right)$ for all $m$. All the utilities will change appropriately to include $\Phi$, for example:

$$
\begin{aligned}
& U_{m}^{\alpha}(\mu,(\Phi, \beta)) \\
& \quad=\mathbb{E}_{\mathbf{h}}\left[\sum_{\mathbf{s}} \min \left\{\phi_{m}(\mathbf{s}), f\left(h_{m}\right)\right\} \mu(\mathbf{s} \mid \mathbf{h}) \beta(m \mid \mathbf{s})\right] .
\end{aligned}
$$

A profile $\left(\mu_{1}^{*}, \cdots, \mu_{M_{1}}^{*},\left(\Phi^{*}, \beta^{*}\right)\right)$ is a NE for G3 if,

$$
\mu_{m}^{*}=\arg \max _{\mu_{m}} U_{m}^{\alpha}\left(\left(\mu_{m}, \mu_{-m}^{*}\right),\left(\Phi^{*}, \beta^{*}\right)\right) \text { for all } m
$$

$\left(\Phi^{*}, \beta^{*}\right)=\arg \max _{(\Phi, \beta)} U_{B S}^{\alpha}\left(\mu^{*},(\Phi, \beta)\right)$.

When BS knows signal statistics, $\left\{p_{\mathbf{s}}\right\}$, it can estimate the ASA utilities for any scheduling policy and for any mobile profile $\mu$ as:

$$
U_{m}^{A S A}(\mu,(\Phi, \beta))=U_{m}^{A S A}\left(p_{\mathbf{s}},(\Phi, \beta)\right):=\mathbb{E}_{\mathbf{s}}[\phi(\mathbf{s}) \beta(m \mid \mathbf{s})] .
$$

In the above the expectation is w.r.t. $p_{\mathbf{s}}$. It can also estimate their cooperative shares $\left\{\theta_{m}^{\alpha c}\right\}$ of (10) using its prior knowledge: the channel statistics. We now propose a robust policy at the BS which uses both these average utilities. The key idea is to design a policy at BS which does not allow the (average) utility of any mobile $m$ to be greater than $\theta_{m}^{\alpha c}$.

When a noncooperative mobile uses a signaling strategy to improve its ATA utility $U_{m}^{A T A}$, even its ASA utility $U_{m}^{A S A}$ improves. The BS can estimate $U_{m}^{A S A}$ of each of the mobiles and hence can sense the increase in the noncooperative mobile's ASA utility in comparison to its cooperative share. The BS can ensure none of the cooperative mobiles is allocated more than its corresponding cooperative share: by allocating only a fraction and not the total signaled utility at every sample. The fraction to be allocated, is set based on the present excess over the cooperative share as follows:

$$
\begin{aligned}
\phi_{m}\left(s_{m}, p_{\mathbf{s}}, \beta\right): & =\left(f\left(s_{m}\right)-\left(U_{m}^{A S A}\left(p_{\mathbf{s}},(\Phi, \beta)\right)-\theta_{m}^{\alpha c}\right) \Delta\right) \\
& 1_{\left\{\left(f\left(s_{m}\right)-\left(U_{m}^{A S A}\left(p_{\mathbf{s}},(\Phi, \beta)\right)-\theta_{m}^{\alpha c}\right) \Delta\right)>0\right\}}
\end{aligned}
$$

for some large value of $\Delta$. Hence, to ensure that none of the mobiles get more ASA utility than its cooperative share, BS needs to allocate (choose $\Phi=\left\{\phi_{m}\right\}$ ) to satisfy the following:

$$
U_{m}^{A S A}\left(p_{\mathbf{s}},(\Phi, \beta)\right)=\mathbb{E}_{\mathbf{s}}\left[\phi_{m}\left(s_{m}, p_{\mathbf{s}}, \beta\right) \beta(m \mid \mathbf{s})\right] .
$$

Both the equations (18) and (17) are satisfied if there exists a fixed point $U_{m}^{A S A}=U_{m}^{A S A}\left(p_{\mathbf{s}}, \beta\right)$ which satisfies:

$$
\begin{aligned}
U_{m}^{A S A} & =\mathbb{E}_{\mathbf{s}}\left[\phi_{m} \beta(m \mid \mathbf{s}) 1_{\left\{\phi_{m}>0\right\}}\right] \\
\phi_{m} & :=f\left(s_{m}\right)-\left(U_{m}^{A S A}-\theta_{m}^{\alpha c}\right) \Delta .
\end{aligned}
$$

With $C_{f}$ representing the upper bound on $f$,

$$
\begin{gathered}
\left(f\left(s_{m}\right)-\left(U_{m}^{A S A}-\theta_{m}^{\alpha c}\right) \Delta\right) 1_{\left\{\left(f\left(s_{m}\right)-\left(U_{m}^{A S A}-\theta_{m}^{\alpha c}\right) \Delta\right)>0\right\}} \\
\leq C_{f}+\theta_{m}^{\alpha c} \Delta \text { for all } s_{m} \text { and } U_{m}^{A S A} .
\end{gathered}
$$

Thus the map $U_{m}^{A S A} \mapsto \phi_{m} \beta(m \mid \mathbf{s}) 1_{\left\{\phi_{m}>0\right\}}$ is bounded and continuous almost surely and hence by bounded convergence theorem the map of (19), $U_{m}^{A S A} \mapsto \mathbb{E}_{\mathbf{s}}\left[\phi_{m} \beta(m \mid \mathbf{s}) 1_{\left\{\phi_{m}>0\right\}}\right]$, is continuous. Thus there exists an $U_{m}^{A S A}$ satisfying the fixed point equation (19) by Brouwer fixed point theorem ${ }^{6}$.

\footnotetext{
${ }^{6}$ Brouwer fixed point theorem: Every continuous function $f$ from a closed ball of a Euclidean space to itself has a fixed point, i.e., an $x^{*}$ which satisfies $x^{*}=f\left(x^{*}\right)$.
} 
With the above allocation, ATA utility gained by mobile $m$,

$$
\begin{aligned}
& U_{m}^{A T A}(\mu,(\Phi, \beta))=\mathbb{E}_{\mathbf{h}, \mathbf{s}}\left[f_{m}^{\text {gain }}\left(h_{m}, s_{m}, p_{\mathbf{s}}, \beta\right) \beta(m \mid \mathbf{s})\right] \\
& \quad f_{m}^{\text {gain }}\left(h_{m}, s_{m}, p_{\mathbf{s}}, \beta\right):=\min \left\{f\left(h_{m}\right), \phi_{m}\left(s_{m}, p_{\mathbf{s}}, \beta\right)\right\} .
\end{aligned}
$$

Equation (19) satisfies :

$$
\begin{aligned}
U_{m}^{A S A} & =\frac{\mathbb{E}_{\mathbf{s}}\left[f\left(s_{m}\right) \beta(m \mid \mathbf{s}) 1_{m}\right]+\theta_{m}^{\alpha c} \Delta \mathbb{E}_{\mathbf{s}}\left[\beta(m \mid \mathbf{s}) 1_{m}\right]}{1+\Delta \mathbb{E}_{\mathbf{s}}\left[\beta(m \mid \mathbf{s}) 1_{m}\right]} \\
\text { with } 1_{m} & :=1_{\left\{\Delta U_{m}^{A S A}<f\left(s_{m}\right)+\Delta \theta_{m}^{\alpha c}\right\}} .
\end{aligned}
$$

Hence, for any strategy profile $(\mu, \beta)$

$$
\begin{aligned}
U_{m}^{A S A} & \left(p_{\mathbf{s}},(\Phi, \beta)\right)-\theta_{m}^{\alpha c} \\
& =\frac{\mathbb{E}_{\mathbf{s}}\left[f\left(s_{m}\right) \beta(m \mid \mathbf{s}) 1_{\left\{\Delta U_{m}^{A S A}<f\left(s_{m}\right)+\Delta \theta_{m}^{\alpha c}\right\}}\right]-\theta_{m}^{\alpha c}}{1+\Delta \mathbb{E}_{\mathbf{s}}\left[\beta(m \mid \mathbf{s}) 1_{\left\{\Delta U_{m}^{A S A}<f\left(s_{m}\right)+\Delta \theta_{m}^{\alpha \alpha}\right\}}\right]} \\
& \leq \frac{C_{f}}{\Delta} \leq o(1 / \Delta),
\end{aligned}
$$

and hence,

$$
U_{m}^{A T A}(\mu,(\Phi, \beta)) \leq U_{m}^{A S A}(\mu,(\Phi, \beta)) \leq \theta_{m}^{\alpha c}+o(1 / \Delta) .
$$

The above is true as, $f_{m}^{\text {gain }}\left(h_{m}, s_{m}, \mu, \beta\right) \leq \phi_{m}\left(s_{m}, \mu, \beta\right)$. In other words, with new allocation (19) at BS, no mobile can gain o $(1 / \Delta)$ more than its cooperative share for any pair $(\mu, \beta)$.

Further, if BS uses any $\alpha$-fair scheduler $\beta^{*}$ of (2), along with allocation policy (19), it is easy to check using (21) and (20) that under truthful strategies (note $\left.p_{\mathbf{s}}=p_{\mathbf{h}}\right) U_{m}^{A S A}\left(\mu^{T}, \beta_{1}^{*}\right)=$ $U_{m}^{A T A}\left(\mu^{T}, \beta_{1}^{*}\right)=\theta_{m}^{\alpha c}$ for all $m$. Also now,

$$
\begin{aligned}
U_{m}^{A S A}\left(p_{\mathbf{h}},\left(\Phi, \beta^{*}\right)\right)-\theta_{m}^{\alpha c} \\
=\frac{\mathbb{E}_{\mathbf{h}}\left[f\left(h_{m}\right) \beta^{*}(m \mid \mathbf{h}) 1_{m}\right]-\mathbb{E}_{\mathbf{s}}\left[f\left(h_{m}\right) \beta^{*}(m \mid \mathbf{h})\right]}{1+\Delta \mathbb{E}_{\mathbf{h}}\left[\beta^{*}(m \mid \mathbf{h}) 1_{m}\right]} \\
=-\frac{\mathbb{E}_{\mathbf{h}}\left[f\left(h_{m}\right) \beta^{*}(m \mid \mathbf{h}) 1_{\left\{\Delta U_{m}^{A S A} \geq f\left(h_{m}\right)+\Delta \theta_{m}^{\alpha c}\right\}}\right]}{1+\Delta \mathbb{E}_{\mathbf{h}}\left[\beta^{*}(m \mid \mathbf{h}) 1_{m}\right]} .
\end{aligned}
$$

The above indicates that $U^{A S A}\left(p_{\mathbf{h}},\left(\Phi, \beta^{*}\right)\right) \leq \theta_{m}^{\alpha c}$. If it was strictly less than the cooperative share, then the indicator in the numerator of the second line can never be true and hence there exists only one fixed point, $\theta_{m}^{\alpha c}$ with $\left(\mu^{T}, \beta^{*}\right)$. We have thus proved:

Theorem 1: If BS knows cooperative shares $\left\{\theta_{m}^{\alpha c}\right\}$ and the signal statistics $\left\{p_{\mathbf{s}}\right\}$, the $M_{1}+1$ player strategic game has an $\epsilon$-NE, i.e., TRE: $\left(\mu^{T},\left(\left\{\phi_{m}\left(s_{m}, p_{\mathbf{s}}, \beta^{*}\right)\right\}, \beta^{*}(m \mid \mathbf{s})\right)\right)$.

In the coming sections, we will turn our attention to iterative algorithms which can achieve a desired level of 'fairness' even in the presence of some noncooperative mobiles. We begin this task by first studying $\alpha$-FSA ([15]).

\section{FAIr SCheduler Algorithm ( $\alpha$-FSA)}

From this section onwards the channel states $\mathbf{h}$ as well as the signaled states $\mathbf{s}$ (the states reported by the mobiles) are continuous random variables with stationary rates across the time, $\left\{r_{m, k}\right\}_{k \geq 1}=\left\{f\left(h_{m, k}\right)\right\}_{k \geq 1},\left\{r_{m, k}^{s}\right\}_{k \geq 1}=\left\{f\left(s_{m, k}\right)\right\}_{k \geq 1}$ for all $m$, satisfying the assumptions of Appendix $\mathrm{A}^{7}$.

This section and the coming section use various types of rates and hence the notations become complicated. Thus a table (in table III) of notations specific to these two sections is given in Appendix A, where all the rate notations are listed at one place.

By assumption A.3 of Appendix A, the rates are integrable and hence the map

$$
\begin{gathered}
\Theta \mapsto\left[\mathbb { E } _ { \mathbf { h } } \left[f\left(h_{1}\right) \beta(1 \mid \mathbf{h}, \Theta), \quad \cdots, \quad \mathbb{E}_{\mathbf{h}}\left[f\left(h_{M}\right) \beta(M \mid \mathbf{h}, \Theta)\right],\right.\right. \\
\beta(m \mid \mathbf{h}, \Theta)=\frac{1_{\left\{m=\arg \max _{j} d \Gamma^{\alpha}\left(\theta_{j}\right) f\left(h_{j}\right)\right\}}}{\left|\left\{\arg \max _{j} d \Gamma^{\alpha}\left(\theta_{j}\right) f\left(h_{j}\right)\right\}\right|}
\end{gathered}
$$

has a fixed point $\bar{\Theta}$ by Brouwer's fixed point theorem and $\beta^{*}(. \mid \mathbf{h}):=\beta(. \mid \mathbf{h}, \bar{\Theta})$ exactly satisfies (3) and hence is a $\alpha$-fair solution. Thus with continuous rates we always have fixed point $\alpha$-fair solution (3). We outlined an algorithm to implement $\alpha$-fair scheduler (3) in Remark II-2 following Lemma 1. The $\alpha$-FSA ([15]), a stochastic approximation based fair scheduling algorithms, exactly follows this outline (with

$$
\begin{aligned}
\left.\Theta_{k}^{\alpha}:=\left[\theta_{1, k}^{\alpha}, \cdots, \theta_{M, k}^{\alpha}\right], \mathbf{r}_{k}:=\left[r_{1, k}, \cdots, r_{M, k}\right]\right): & \\
\theta_{m, k}^{\alpha} & =\theta_{m, k-1}^{\alpha}+\epsilon_{k}\left[I_{m}^{\alpha}\left(\mathbf{r}_{k}, \Theta_{k-1}^{\alpha}\right) r_{m, k}-\theta_{m, k-1}^{\alpha}\right] \\
I_{m}^{\alpha}(\mathbf{r}, \Theta) & =1_{\left\{m=\arg \max _{j} d \Gamma^{\alpha}\left(d_{j}+\theta_{j}\right) r_{j}\right\}} \\
& =1_{\left\{m=\arg \max _{j} r_{j}\left(\theta_{j}+d_{j}\right)^{-\alpha}\right\}}
\end{aligned}
$$

where $d_{m}$ are small positive constants (added for stability). While making decisions $\left\{I_{m}^{\alpha}\right\}$, if there are more than one users attaining maximum, one of the maximizers is chosen by the BS randomly. In [15, Th. 2.2], the authors show that $\left\{\theta_{m, k}^{\alpha}\right\}$ of (22), with $\alpha \leq 1$, converges weakly to the unique limit point $\Theta^{*}$ that satisfies $\mathbb{E}\left[r_{m} I_{m}^{\alpha}\left(\mathbf{r}, \Theta^{*}\right)\right]=\theta_{m}^{*}$ for all $m$. A close look at this limit point (when we neglect $\left\{d_{m}\right\}$ ) reveals that $I_{m}^{\alpha}\left(\mathbf{r}, \Theta^{*}\right)$ is the $\alpha$-fair scheduler (3) and that $\Theta^{*}$ are the unique cooperative shares, $\left\{\theta_{m}\left(\beta^{*}\right)\right\}=\left\{\theta_{m}^{c \alpha}\right\}$. Thus, $\alpha-F S A$ weakly converges to the unique point (cooperative shares) that maximizes the $\alpha$-fair criterion (1).

\section{A. Convergence of $\alpha-F S A$ in presence of noncooperation}

The $\alpha$-FSA uses signaled rates, $r_{m, k}^{s}:=f\left(s_{m, k}\right)$ and $\mathbf{r}_{k}^{s}=$ $\left[r_{1, k}^{s}, \cdots, r_{M, k}^{s}\right]^{t}$ to make decisions, as in Section IV:

$$
\theta_{m, k}^{\alpha}=\theta_{m, k-1}^{\alpha}+\epsilon_{k}\left[I_{m}^{\alpha}\left(\mathbf{r}_{k}^{s}, \Theta_{k-1}^{\alpha}\right) r_{m, k}^{s}-\theta_{m, k-1}^{\alpha}\right] .
$$

These signaled rates reflect the statistics $p_{\mathbf{s}}$ (instead of $p_{\mathbf{h}}$ ), there again is weak convergence, however this time to a different attractor corresponding to $p_{\mathbf{s}}$. It is very easy to see as in Section IV that, when mobiles are noncooperative with

\footnotetext{
${ }^{7}$ For understanding the asymptotic limits of the dynamic algorithms of this section we will need the results corresponding to the static settings of Section II. But, all the results of Section II correspond to discrete channel states and rates. We assume that even for the more general case under study in this section, an $\alpha$-fair solution of the form (3) exists and that the corresponding shares $\left\{\theta_{m}^{\alpha c}\right\}$ are unique as in Lemma 1. Sufficient conditions for this to occur are under study. This result is required for showing that $\alpha$-FSA asymptotically converges to the cooperative shares (i.e., limits maximize the $\alpha$-fair criterion) for all $\alpha$. In [15] Theorem 2.3 does this job approximately at least for $\alpha \leq 1$ : any other assignment rule results in a limit $\Theta$ with $\sum_{m} \Gamma^{\alpha}\left(\theta_{m}\right)$ less than that corresponding to scheduler $\left\{I_{m}^{\alpha}\right\}$ of $\alpha$-FSA (22). The simulations of this section also confirm the results we obtained based on this assumption.
} 
profile $\mu, \alpha-F S A$ converges weakly to unique maximum ASA rates, $\left\{U_{m}^{A S A}\left(\mu, \beta_{\mu}^{*}\right)\right\}$ with $\beta_{\mu}^{*}$ defined by (12).

\section{B. Failure of $\alpha-F S A$ in presence of noncooperation}

As noted above, the $\alpha$-FSA (22) converges to the maximum ASA utility (under $\mu$ ) which need not be equal to the ATA utility, in the presence of noncooperation. However, to understand the behavior of (22) in presence of noncooperation, one needs to study the asymptotic true utilities gained by the mobiles under (22). Towards this, we consider a second iteration running in parallel with (22), with only the instantaneous signaled utility $r_{m, k}^{s}$ replaced by the true instantaneous utility obtained by the mobile, $\bar{r}_{m, k}:=\min \left\{r_{m, k}, r_{m, k}^{s}\right\}$ :

$$
\bar{\theta}_{m, k}^{\alpha}=\bar{\theta}_{m, k-1}^{\alpha}+\epsilon_{k}\left[I_{m}^{\alpha}\left(\mathbf{r}_{k}^{s}, \Theta_{k-1}^{\alpha}\right) \bar{r}_{m, k}-\bar{\theta}_{m, k-1}^{\alpha}\right]
$$

As in [15], one can show that $\bar{\theta}_{m, k}$ converges weakly to $U_{m}^{A T A}\left(\mu, \beta_{\mu}^{*}\right)$, the ATA utility under $\left(\mu, \beta_{\mu}^{*}\right)$.

Thus, the asymptotic limits of $\alpha$-FSA equal maximum ASA utilities of section IV while the true utility adaptation (23) converges to the corresponding ATA utilities. These time limits will thus have all the properties of section IV: the $\alpha$-FSA will fail for small $\alpha$ and will be robust for large $\alpha$ as discussed in section IV. The only difference here is that the channel rates are continuous.

\section{Numerical examples}

Two asymmetric users are considered in Figure 3. Let $Z\left(\sigma^{2}\right)$ be a Rayleigh random variable with density $f_{Z}\left(z ; \sigma^{2}\right)=$ $z e^{-z^{2} / 2 \sigma^{2}}$. Channel state of user 1 is conditional Rayleigh distributed, i.e.,

$$
h_{1} \sim \frac{f_{Z}(z ; 1) 1_{\{z \leq 2\}} d z}{P(Z(1) \leq 2)} .
$$

User 2 has almost a constant channel,

$$
h_{2}-0.45 \sim \frac{f_{Z}(z ; 0.05) 1_{\{z \leq 2\}} d z}{P(Z(0.05) \leq 2)} .
$$

The utilities are the achievable rates $f(h)=\log (1+h)$. User 1 is noncooperative with $s_{1}(h)=h(1-\delta)+2 \delta$ with $\delta=0.9$. We plot the limit of the $\alpha$-FSA, the limits of true utility adaptation (23) as function ${ }^{8}$ of $\alpha$. We also plot the cooperative shares, obtained by the limits of $\alpha$-FSA, i.e., with $\delta=0$. We observe that the cooperative shares tend towards equal values as $\alpha$ tends to infinity. User 1 is successful in gaining more utility in comparison with its cooperative share for all $\alpha$ less than 1.2. Beyond 1.2, user 1 actually loses and the loss increases as $\alpha$ increases. The observations are similar to that in motivating example and indicate that $\alpha$-FSA is robust only for large $\alpha$.

In table I, we consider a symmetric example. In this example, we consider the discrete channels of section IV. We could have constructed examples with symmetric continuous channel states as in previous example and demonstrate the failure of $\alpha$-FSA. But we note that the $\alpha$-FSA even with discrete rates

\footnotetext{
${ }^{8}$ The author in [15] analyze these algorithms only for $\alpha \leq 1$. However we confirm using numerous examples that they work in fact for all values of $\alpha$, i.e., when all mobiles are cooperative the $\alpha-\mathrm{FSA}$ for all $\alpha$ converge to the unique shares which maximize the objective function (4).
}

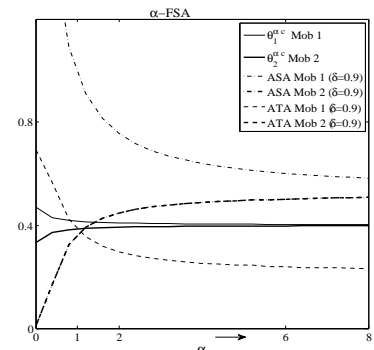

Fig. 3. $\alpha$-FSA : Maximum ASA and corresponding ATA shares versus $\alpha$.

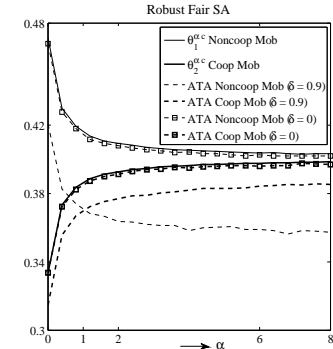

Fig. 4. Robust Policy: Maximal ASA and corresponding ATA shares. works as explained in this section and hence this example is given to demonstrate the same. We consider two users, both of them having two channel states with utilities $a 1=4$, $a 2=2$ occurring with probabilities $p 1=0.3, p_{2}=0.7$ respectively. In this example we work only with $\alpha=1$, i.e., the proportional fair scheduler. Both users have equal cooperative share, $\theta_{1}\left(\beta^{*}\right)=\theta_{2}\left(\beta^{*}\right)=1.51$. Hence when both the mobiles report the channel states truthfully, under $\alpha-\mathrm{FSA}$ scheduler, the asymptotic throughputs of both the mobiles converge to 1.51 , i.e., $\lim _{k \rightarrow \infty} \theta_{m, k}=1.51$ for $m=1,2$. Hence maximum proportionally fair BS (asymptotic) utility is $U_{B S}^{*}=2 \log (1.51)=0.824$.

\begin{tabular}{|c|c|c|c|}
\hline$\mu_{1}\left(a_{1} \mid a_{2}\right)$ & True Rates & & \\
$t$ & $\left(U_{1}^{A T A}, U_{2}^{A T A}\right)$ & $\sum_{m} U_{m}^{A T A}$ & $\sum_{m} \log \left(U_{m}^{A T A}\right)$ \\
\hline 0 (Coop) & $(1.51,1.51)$ & 3.02 & 0.824 \\
\hline 0.8 & $(1.62,1.39)$ & 3.01 & 0.812 \\
\hline 0.9 & $(1.72,1.3)$ & 3.02 & 0.804 \\
\hline 0.98 & $(1.70,1.3)$ & 3.0 & 0.793 \\
\hline
\end{tabular}

TABLE I

A SYMMETRIC EXAMPLE IN WHICH $\alpha$-FSA FAILS AGAINST NONCOOPERATION

The user 1 becomes noncooperative with $\mu_{1}\left(a_{1} \mid a_{2}\right)=t$. We see that the user 1 is successful in grabbing the channel more often and increasing its utility in comparison with its cooperative share. The more he cheats (the more $t$ is) the more he gains (look at the asymptotic throughput $U_{1}^{A T A}$, given in the second column in table I). He gains up to $12.5 \%$ more than its cooperative share. The cooperative user, user 2 has lost due to the non cooperative mobile resulting in unfair allocations.

\section{RoBUST $\alpha$-FAIR Algorithms : RoBUSt FAIR SA}

We saw that $\alpha$-FSA fails in the presence of noncooperative users. Hence, we propose a robustification of $\alpha$-FSA against noncooperation using the policies of subsection V-B. In V-B, we proposed BS policies robust against noncooperation and in this section we propose stochastic approximation based algorithms to converge towards the ASA utilities of those policies given by (20). The policy of section V-B requires the knowledge of signal statistics $p_{\mathbf{s}}$, which has to be estimated. Basically the methods described in this section (as is done by $\alpha-\mathrm{FSA}$ ) combine the estimation and control using stochastic 
approximation based methods. We will show robustness of these policies by using appropriate game theoretic tools.

Robust Policy 1 : We now propose a robustification of (22) against noncooperation in the following :

$$
\begin{aligned}
\theta_{m, k+1}^{\alpha} & =\theta_{m, k}^{\alpha}+\epsilon_{k}\left[\phi_{m, k+1}^{\alpha} I_{m}^{\alpha}\left(\mathbf{r}_{k+1}^{s}, \Theta_{k}^{\alpha}\right)-\theta_{m, k}^{\alpha}\right] \\
\phi_{m, k+1}^{\alpha} & =\max \left\{0,\left(r_{m, k+1}^{s}-\left(\theta_{m, k}^{\alpha}-\theta_{m}^{\alpha c}\right) \Delta\right)\right\},(24) \\
\theta_{m, 0}^{\alpha} & =\theta_{m}^{\alpha c}
\end{aligned}
$$

where the decisions $I_{m}^{\alpha}(\mathbf{r}, \Theta)$ are same as that in $\alpha$-FSA (22) and only the allocations $\Phi_{k}^{\alpha}:=\left[\phi_{1, k}^{\alpha}, \cdots, \phi_{M, k}^{\alpha}\right]^{T}$ are made robust. As in the case of $\alpha$-FSA, to understand the behavior of this algorithm we need the following iteration which estimates the true utilities gained by the mobiles :

$$
\begin{aligned}
\hat{\theta}_{m, k+1}^{\alpha} & =\hat{\theta}_{m, k}^{\alpha}+\epsilon_{k}\left[\hat{r}_{m, k+1}^{\alpha} I_{m}^{\alpha}\left(\mathbf{r}_{k+1}^{s}, \Theta_{k}^{\alpha}\right)-\hat{\theta}_{m, k}^{\alpha}\right] \\
\hat{r}_{m, k+1}^{\alpha} & =\min \left\{r_{m, k+1}, \quad \phi_{m, k+1}^{\alpha}\right\}
\end{aligned}
$$

\section{A. Analysis :}

We analyze the robustness of the proposed algorithm using game theoretical tools. Fix any $\alpha$. We consider a $M_{1}+1$ player game with utilities defined by :

$$
U_{m}:=\lim _{k \rightarrow \infty} \hat{\theta}_{m, k+1}^{\alpha} \text { for all } m \text { and } U_{B S}:=\sum_{m} \Gamma^{\alpha}\left(U_{m}\right) .
$$

We analyze the limits of (26) using ODE approximation methods (for e.g., [15], [9]). As a first step, we obtain the following ODE approximation result .

Theorem 2: Assume that algorithms (24), (26) satisfy assumptions A.1, A.2, and A.3 of Appendix A . For any initial conditions, $\left(\Theta_{k}^{\alpha}, \hat{\Theta}_{k}^{\alpha}\right)$ converge weakly to the set of limit points of the solution of the ODE (for all $m \leq M$ ):

$$
\begin{aligned}
& \dot{\theta_{m}}=\bar{h}_{m}(\Theta)-\theta_{m}, \quad \bar{h}_{m}(\Theta)=\mathbb{E}\left[\phi_{m}^{\alpha} I_{m}^{\alpha}\left(\mathbf{r}^{s}, \Theta\right)\right], \\
& \dot{\hat{\theta}}_{m}=\overline{\hat{h}}_{m}(\Theta)-\hat{\theta}_{m}, \quad \overline{\hat{h}}_{m}(\Theta)=\mathbb{E}\left[\hat{r}_{m}^{\alpha} I_{m}^{\alpha}\left(\mathbf{r}^{s}, \Theta\right)\right] .
\end{aligned}
$$

These conclusions hold whenever $\epsilon_{n} \rightarrow 0, \sum_{n} \epsilon_{n}=\infty$ and for some $\alpha_{n} \rightarrow \infty, \lim _{n} \sup _{0 \leq l \leq \alpha_{n}}\left|\epsilon_{n+l} / \epsilon_{n}-1\right|=0$.

Remarks about the proof and the assumptions : This theorem can be proved exactly in the same way as is done for $\alpha$-FSA by Theorem 2.1 of Kushner et al's [15]. The required assumptions A.1-3 are also very similar to that in [15] and these are satisfied in almost the same conditions as mentioned in [15].

Hence, one can upper bound utilities $\left\{U_{m}\right\}$ by upper bounding all the attractors of ODE (28). Any attractor $\Theta^{*}$ of the ODE (27) satisfies

$$
\begin{aligned}
& \theta_{m}^{*}-\theta_{m}^{\alpha c}=\frac{\mathbb{E}\left[r_{m}^{s} I_{m}^{\alpha}\left(\mathbf{r}^{s}, \Theta^{*}\right) I_{\left\{\phi_{m}^{\alpha}>0\right\}}\right]-\theta_{m}^{\alpha c}}{1+\Delta \mathbb{E}\left[I_{m}^{\alpha}\left(\mathbf{r}^{s}, \Theta^{*}\right) I_{\left\{\phi_{m}^{\alpha}>0\right\}}\right]} . \text { Hence } \\
& \theta_{m}^{*}-\theta_{m}^{\alpha c} \leq \frac{C_{f} \mathbb{E}\left[I_{m}^{\alpha}\left(\mathbf{r}^{s}, \Theta^{*}\right) I_{\left\{\phi_{m}^{\alpha}>0\right\}}\right]}{\Delta \mathbb{E}\left[I_{m}^{\alpha}\left(\mathbf{r}^{s}, \Theta^{*}\right) I_{\left\{\phi_{m}^{\alpha}>0\right\}}\right]}=\frac{C_{f}}{\Delta}
\end{aligned}
$$

where $C_{f}$ is the upper bound on signaled rates $\left\{r_{m}^{s}\right\}$. Thus, $\theta_{m}^{*} \leq \theta_{m}^{\alpha c}+o(1 / \Delta)$. Further, any attractor of ODE (28) satisfies $\hat{\theta}_{m}^{*}=\hat{h}_{m}\left(\Theta^{*}\right)$ leading to $\hat{\theta}_{m}^{*} \leq \theta_{m}^{*}$. Thus for any mobile strategy profile $\mu$,

$$
U_{m} \stackrel{w}{=} \hat{\theta}_{m}^{*} \leq \theta_{m}^{*} \leq \theta_{m}^{\alpha c}+o(1 / \Delta)
$$

So, none of the users, no matter what strategy they use or the others use, can gain more than $\theta_{m}^{\alpha c}$.

Under $\mu^{T}, \Theta^{\alpha c}=\left[\theta_{1}^{\alpha c}, \cdots, \theta_{M}^{\alpha c}\right]^{T}$ is the only zero of RHS of both the ODEs (27), (28) as shown using fixed point analysis in section V-B (note here that $I_{m}^{\alpha}\left(\mathbf{r}^{s}, \Theta\right)$ is the $\alpha$ fair scheduler $\beta^{*}(\mid \mathbf{s})$ satisfying (3)). By virtue of Lemma 4, one can easily show that it will indeed be an attractor (for large enough values of $\Delta$ ) for ODE (27) by showing that the derivative of $\left\{\bar{h}_{m}(\Theta)-\theta_{m} ; m \leq M\right\}$ is negative definite ${ }^{9}$ near $\Theta^{\alpha c}$. And then it is immediate that $\Theta^{\alpha c}$ is also an attractor for the ODE (28). Thus, $\Theta^{\alpha c}$, is the only attractor of both the ODEs under $\mu^{T}$. Thus

$$
U_{m} \stackrel{w}{=} \theta_{m}^{\alpha c} \text { for all } m \text { under } \mu^{T} .
$$

From (29), (30), the robust policy (24) at BS together with the truth-revealing policy of users forms an $\epsilon-N E$.

Robust Policy 2 : The policies of previous subsection, Robust Policy 1 will not allow the ATA utility of any user to go above the cooperative share. Nevertheless, when a user is noncooperative, these policies may still result in a loss for the cooperative users: the noncooperative user can still grab the channel from other users, without getting a gain because of the robust allocation policies (19). To avoid this problem, we may robustify the decisions as well:

$$
\theta_{m, k+1}^{\alpha}=\theta_{m, k}^{\alpha}+\epsilon_{k}\left[\phi_{m, k+1}^{\alpha} I_{m}^{\alpha}\left(\Phi_{k+1}^{\alpha}, \Theta_{k}^{\alpha}\right)-\theta_{m, k}^{\alpha}\right] .
$$

The analysis of this policy would be similar to the policy 1. We need to change the assumptions of the Appendix A appropriately (need to replace the decisions $I_{m}^{\alpha}\left(\mathbf{r}^{s}, \Theta\right)$ with $I_{m}^{\alpha}\left(\Phi^{\alpha}, \Theta\right)$ in all the places) for ODE approximation. However these policies are more complicated and hence further analysis is more difficult. We will still be able to go through all steps in the analysis exactly in a similar way, except that we will not be able to show the uniqueness of the attractor under truthful strategies $\mu^{T}$. However, we could ensure the robustness of these policies using the numerical examples given below. The examples also show that these policies outperform Robust

${ }^{9}$ Using similar steps as those used for deriving (34) of Appendix B, one can easily see that

$$
\begin{aligned}
& \left.\frac{\partial\left(\bar{h}_{m}(\Theta)-\theta_{m}\right)}{\partial \theta_{m}}\right|_{\Theta=\Theta^{\alpha c}}=-1-\Delta E_{\mathbf{s}}\left[I_{m}^{\alpha}\left(\mathbf{r}^{s}, \Theta^{\alpha c}\right)\right] \\
& -\alpha E_{\mathbf{s}}\left[\left(r_{m}^{s}\right)^{2} \sum_{j \neq m} \Pi_{k \neq j, i} \operatorname{Pr}\left(A_{k}\left(r_{m}^{s}, \Theta^{\alpha c}\right)\right) \frac{g_{s_{j}}(\kappa)\left(\theta_{j}^{\alpha c}+d_{j}\right)^{\alpha}}{\left(\theta_{m}^{\alpha c}+d_{m}\right)^{\alpha+1}}\right]
\end{aligned}
$$

which is always negative and whose magnitude increases as $\Delta$ increases for all $m$ while for any $m \neq j$

$$
\begin{gathered}
\left.\frac{\partial\left(\bar{h}_{m}(\Theta)-\theta_{m}\right)}{\partial \theta_{j}}\right|_{\Theta=\Theta^{\alpha c}}= \\
\alpha E_{\mathbf{s}}\left[\left(r_{m}^{s}\right)^{2} \Pi_{k \neq j, i} \operatorname{Pr}\left(A_{k}\left(r_{m}^{s}, \Theta^{\alpha c}\right)\right) \frac{g_{s_{j}}(\kappa)\left(\theta_{j}^{\alpha c}+d_{j}\right)^{\alpha-1}}{\left(\theta_{m}^{\alpha c}+d_{m}\right)^{\alpha}}\right]
\end{gathered}
$$

whose magnitude is bounded independent of $\Delta$. Define the map $\bar{H}(\Theta):=$ $\left[\bar{h}_{1}(\Theta), \cdots, \bar{h}_{M}(\Theta)\right]^{T}$, the total derivative w.r.t $\Theta A:=D_{\Theta}(\bar{H}(\Theta)-\Theta)$ and the matrix $B$ as a diagonal matrix consisting of only diagonal entires of matrix $A$. Note that matrix $B$ has all negative eigenvalues. Now, when [10, Corrollary III 2.6, pp. 63] is applied to matrices $A, B$ (this corollary compares the eigenvalues of the two matrices) we get that matrix $A$ becomes negative definite as the value of $\Delta$ increases. 
policy 1 in many ways, while Robust policy 1 would be simpler to implement.

\section{Numerical examples}

We continue with the example of Figure 3 (in which $\alpha$ FSA failed) in Figure 4. We use Robust Fair SA Policy 1 in place of $\alpha$-FSA. We set $\Delta=1000$. We plot only the ATA utilities for both values of $\delta=0, \delta=0.9$. We do not plot the ASA utilities in this figure as these utilities for all the cases are very close to cooperative shares $\Theta^{\alpha c}$. We see that this policy is indeed robust : 1) the time limits of $\left\{\theta_{m, k}\right\}$ (which correspond to ASA utilities) are very close to the cooperative shares; 2) the time limit of the asymptotic true (ATA) utilities are lesser than the cooperative shares for the noncooperative mobile. It is also lesser for cooperative mobile, but the gap between the cooperative shares and the ATA utilities is much lesser for a cooperative mobile (plots corresponding to $\delta=0.9$ ); 3) when all the mobiles are cooperative both the ASA as well as ATA utilities are close to the cooperative shares for all the mobiles (plots corresponding to $\delta=0$ ).

In Figures 5, 6 we compare the two robust policies. Here

$$
h_{1} \sim \frac{f_{Z}(z ; 1) 1_{\{z \leq 2\}} d z}{\operatorname{Prob}(Z(1) \leq 2)}, \quad h_{2} \sim \frac{f_{Z}(z ; 0.5) 1_{\{z \leq 2\}} d z}{\operatorname{Prob}(Z(0.5) \leq 2)},
$$

$f(h)=\log (1+h)$ and $\Delta=1000$. Mobile 1 , can be noncooperative using $s_{1}(h)=h+(2-h) \delta$ with $\delta=0.9$. We see from the figures that both the policies are robust. Even with high values of $\delta=0.9$ (which indicates large amount of noncooperation) both the policies do not allow the ATA utilities to go beyond the cooperative shares. However the policy 2 is way better than the policy 1 as: 1) the noncooperative mobile in fact is punished by policy 2, its ATA utility is lesser than the cooperative share $\theta_{1}^{\alpha c}$ (Figure 6), but the same is not true for policy 1 (Figure 5). 2) the cooperative mobile loses because of the noncooperation from the other mobile to much greater extent in policy 1 . This goes in line with the extra robustification built into the decision making by policy 2 . When $\mathrm{BS}$ uses policy 1 , the noncooperative mobile is successful in grabbing the channel (almost always with large values of $\delta=0.9$ ), however will not be able to gain much from it because of the robust allocation (19)-(21). When mobile is aware that he cant gain from being noncooperative, he will as well have to stick to truthful signaling, unless his intentions are that of jamming the other mobile (in which case the BS needs to use policy 2). However the policy 1 is easier to implement than the policy 2 because of simpler decisions and may also have faster convergence.

\begin{tabular}{|c|c|c|c|}
\hline $\begin{array}{c}\mu_{1}\left(a_{1} \mid a_{2}\right)= \\
t\end{array}$ & $\begin{array}{c}\text { True Rates } \\
\left(U_{1}^{A T A}, U_{2}^{A T A}\right)\end{array}$ & $\sum_{m} U_{m}^{A T A}$ & $\begin{array}{c}U_{B S}= \\
\log \left(U_{m}^{A T A}\right)\end{array}$ \\
\hline 0 (Coop) & $(1.51,1.51)$ & 3.02 & 0.824 \\
\hline 0.8 & $(1.30,1.41)$ & 2.7 & 0.606 \\
\hline 0.9 & $(1.31,1.37)$ & 2.68 & 0.585 \\
\hline 0.98 & $(1.32,1.36)$ & 2.68 & 0.585 \\
\hline
\end{tabular}

TABLE II

RoBUSt POLICY 1 AgAINST NONCOOPERATION EXAMPLE OF TABLE I

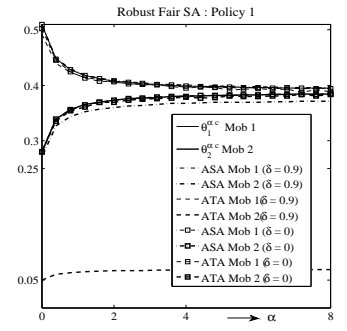
ASA and corresponding ATA shares versus $\alpha$.
Fig. 5. Robust Policy 1 : Maximal

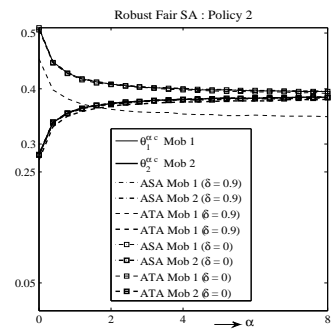

Fig. 6. Robust Policy 2 : Maximal ASA and corresponding ATA shares versus $\alpha$.
In table II we continue with the symmetric example of table I wherein the $\alpha-$ FSA fails. We see once again that (even with discrete and symmetric conditions) the Robust Fair SA policy 1 is robust against noncooperation, it does not allow the noncooperative user to improve its asymptotic throughput.

\section{CONCLUSIONS}

We studied centralized downlink transmissions in a cellular network in the presence of noncooperative mobiles. Using $\alpha$ fair scheduler, the BS has to assign the slot to one of the many mobiles based on truthful information from mobiles about their time-varying channel gains. A noncooperative mobile may misrepresent its signal to the BS so as to maximize his throughput. We modeled a noncooperative mobile as a rational player who wishes to maximize his throughput. For this game, we identified several scenarios related to the awareness of $\mathrm{BS}$. When the BS is unaware of this noncooperative behavior, we model this game as hierarchical game with two levels. We identify that, the presence of noncooperative users, results in an $\alpha$-fair bias in the channel assignment for small values of $\alpha$. As $\alpha$ increases, an $\alpha$-fair scheduler becomes more and more robust to noncooperation irrespective of the awareness of BS and a max-min fair scheduler is always robust. When the BS is aware of the noncooperative mobiles, we characterized a babbling equilibrium which is obtained when both the BS and the noncooperative players make no use of the signaling opportunities. This game has no TRE (Truth Revealing Equilibrium). Using additional knowledge of the statistics of the signals observed at the BS, we built new robust policies to elicit the truthful signals from mobiles and achieve a Truth Revealing Equilibrium. We then studied the popular, iterative fair scheduling algorithm (which we called $\alpha$-FSA) analyzed by Kushner and Whiting in [15]. We showed that $\alpha$ FSA fail under noncooperation. Finally, we proposed iterative robust fair sharing to robustify the $\alpha$-FSA in the presence of noncooperation.

\section{Acknowledgments}

This project was supported by the Indo-French Center for the Promotion of Advanced Research (IFCPAR), project 4000IT-1 and by the INRIA association program DAWN. The french co-authors have also been supported by the Bionets European project and the RNRT-ANR WiNEM project. 


\section{REFERENCES}

[1] Qualcomm, Inc., "1xEV:1x Evolution IS-856 TIA/EIA Standard Airlink Overview", Nov. 2001.

[2] 3GPP TS 25.308, Technical Specification 3rd Generation Partnership Project; Technical Specification Group Radio Access Network; High Speed Downlink Packet Access (HSDPA); Overall description; Stage 2, Release 8, V8.3.0, sept 2008.

[3] R. Agrawal, A. Bedekar, R. J. La, and V. Subramanian, "Class and channel condition based weighted proportional fair scheduler," in Teletraffic Engineering in the Internet Era, Proc. ITC-17, S. da Bahia, J. M. de Souza, N. L. S. da Fonseca, and E. A. de Souza e Silva, Eds. Amsterdam, The Netherlands: North-Holland, 2001, pp. 553565.

[4] E. Altman, J. Galtier, "Generalized Nash Bargaining Solution for bandwidth allocation", COMNET 50, pp 3242-3263, 2006.

[5] R. J. Aumann, "Subjectivity and correlation in randomized strategies" Journal of Mathematical Economics 1 (1974) 67-96.

[6] C. Touati, E. Altman, J. Galtier, "Fair power and transmission rate control in wireless networks", GLOBECOM '02., Nov. 2002, pp. $1229-1233$ vol.2.

[7] D. M. Andrews, K. Kumaran, K. Ramanan, A. L. Stolyar, R. Vijayakumar, and P. A. Whiting, "Scheduling in a queueing system with asynchronously varying service rates," Prob. Eng. Inf. Sci., vol. 18, pp. 191217, 2004.

[8] P. Bender, P. Black, M. Grob, R. Padovani, N. Sindhushayana, and A. Viterbi, "CDMA/HDR: A bandwidth-efficient high-speed wireless data service for nomadic mobiles," IEEE Commun. Mag., vol. 38, no. 7, pp. 7077, July 2000.

[9] A. Benveniste, M. Mietivier and P. Priouret, "Adaptive algorithms and stochastic approximation", Springer-Verlag, April 1990.

[10] R. Bhatia, "Matrix analysis", volume 169 of Graduate Texts in Mathematics, 1997, Springer-Verlag, New York

[11] S. C. Borst and P. A. Whiting, "Dynamic rate control algorithms for HDR throughput optimization," in Proc. IEEE INFOCOM, 2001, pp. 976985.

[12] E. F. Chaponniere, P. J. Black, J. M. Holtzman, and D. N. C. Tse, "Transmitter Directed code division multiple access system using path diversity to equitably maximize throughput," U.S. Patent 6,449,490, Sep. 10, 2002.

[13] V. Kavitha, E. Altman, R. El-Azouzi and R.Sundaresan "Opportunistic scheduling in cellular systems in the presence of non-cooperative mobiles", CDC 2009

[14] V. Kavitha, E. Altman, R. El-Azouzi and R.Sundaresan, "Opportunistic scheduling in cellular systems in the presence of non-cooperative mobiles", Submitted to IEEE Trans. on Information theory.

[15] H.J. Kushner, P.A. Whiting, "Convergence of Proportional-Fair Sharing algorithms under general conditions," IEEE Trans. Wireless Commun. vol. 3, no. 4, pp. 12501259, Jul. 2004.

[16] V. K. N. Lau, "Proportional Fair SpaceTime Scheduling for Wireless Communications", IEEE Trans. communications, vol. 53, no. 8, Aug 2005.

[17] X. Liu, E. K. P. Chong, and N. B. Shroff, "A framework for opportunistic scheduling in wireless networks," Comput. Netw., vol. 41, pp. 451474, 2003.

[18] J. Mo and J. Walrand, "Fair end-to-end window-based congestion control", in Proc. of SPIE International Symposium on Voice, Video and Data Communications, 1998.

[19] J.B. Rosen, Existence and Uniqueness of Equilibrium Points for Concave N-Person Games, Econometrica, 33, 520-534, July 1965.

[20] S. Shakkottai and A. L. Stolyar, "Scheduling algorithms for a mixture of real-time and non-real-time data in HDR," in Teletraffic Engineering in the Internet Era, Proc. ITC-17, S. da Bahia, J. M. de Souza, N. L. S. da Fonseca, and E. A. de Souza e Silva, Eds. Amsterdam, The Netherlands: North-Holland, 2001, pp. 793804.

[21] A.L.Stolyar, "On the asymptotic optimality of the gradient scheduling algorithm for multiuser throughput allocation", Operations Research, vol. 53, no.1, Jan-Feb 2005, pp. 12-15.

[22] Joel Sobel, "Signaling Games, To appear in Encyclopedia of Complexity and System Science", M. Sotomayor (ed.), Springer, forthcoming, 2009.

\section{APPENDIX A : ASSUMPTIONS}

We first reintroduce some of the notations. This table lists all the various rates used in the sections VI, VII and their description. The last column of this table also provides the corresponding vector notation which basically represents the vector of all the $M$ components.

\begin{tabular}{|l|l|c|}
\hline Variable & Description & Vector \\
\hline$r_{m, k}=f\left(h_{m, k}\right)$ & $\begin{array}{l}\text { True rate of } \\
\text { mobile } m \text { at time } k\end{array}$ & $\mathbf{r}_{k}$ \\
\hline$r_{m, k}^{s}=f\left(s_{m, k}\right)$ & $\begin{array}{l}\text { Rate signaled by } \\
\text { mobile } m \text { at time } k\end{array}$ & $\mathbf{r}_{k}^{s}$ \\
\hline $\begin{array}{l}\bar{r}_{m, k}= \\
\min \left\{r_{m, k}, r_{m, k}^{s}\right\}\end{array}$ & $\begin{array}{l}\text { True rate obtained by } \\
\text { mobile } m \text { at time } k \\
\text { under } \alpha \text {-FSA }\end{array}$ & \\
\hline $\begin{array}{l}\phi_{m, k}^{\alpha}= \\
\max \left\{0,\left(r_{m, k}^{s}-\left(\theta_{m, k-1}^{\alpha}-\theta_{m}^{\alpha c}\right) \Delta\right)\right\}\end{array}$ & $\begin{array}{l}\text { Allocated rate by } \\
\text { Robust fair SA to } \\
\text { mobile } m \text { at time } k\end{array}$ & $\Phi_{k}^{\alpha}$ \\
\hline $\begin{array}{r}\text { True rate obtained by } \\
\min \left\{r_{m, k}, \phi_{m, k}^{\alpha}\right\}\end{array}$ & $\begin{array}{l}\text { mobile } m \text { at time } k \\
\text { under Robust fair SA }\end{array}$ & \\
\hline
\end{tabular}

TABLE III

TABLE OF NOTATIONS FOR DIFFERENT RATES

We now state the assumptions required for sections VI, VII.

A.1 Let $\zeta_{k}$ denote, $\left\{\left(\mathbf{r}_{l}, \mathbf{r}_{l}^{s}\right): l \leq k\right\}$, the past. For each $i, k, \zeta_{k}$,

$$
\begin{aligned}
& h_{m, k}\left(\Theta, \zeta_{k}\right):=\mathbb{E}_{k}\left[\phi_{m, k+1}^{\alpha} I_{m}^{\alpha}\left(\mathbf{r}_{k+1}^{s}, \Theta\right)\right], \\
& \hat{h}_{m, k}\left(\Theta, \zeta_{k}\right):=\mathbb{E}_{k}\left[\hat{r}_{m, k+1}^{\alpha} I_{m}^{\alpha}\left(\mathbf{r}_{k+1}^{s}, \Theta\right)\right],
\end{aligned}
$$

are continuous in $\Theta \in R_{+}^{M}$. Here $\Theta$ is considered fixed. Let $\delta>0$ be arbitrary. The continuity is uniform in $k$ and in $\zeta_{k}$ in the set $\left\{\Theta: \theta_{i} \geq \delta, i \leq M\right\}$.

A.2 The sequence $\left\{\left(\mathbf{r}_{l}, \mathbf{r}_{l}^{s}\right): l \leq \infty\right\}$ is stationary. Define the following stationary expectations:

$$
\begin{aligned}
& \bar{h}_{m}(\Theta)=\mathbb{E}\left[\phi_{m, 1}^{\alpha} I_{m}^{\alpha}\left(\mathbf{r}_{1}^{s}, \Theta\right)\right], \\
& \overline{\hat{h}}_{m}(\Theta)=\mathbb{E}\left[\hat{r}_{m, 1}^{\alpha} I_{m}^{\alpha}\left(\mathbf{r}_{1}^{s}, \Theta\right)\right]
\end{aligned}
$$

In the above $\Theta$ is considered fixed. Also,

$$
\begin{aligned}
& \lim _{k, n \rightarrow \infty} \frac{1}{k} \sum_{l=n}^{n+k-1}\left[\mathbb{E}_{n}\left[\phi_{m, l+1}^{\alpha} I_{m}^{\alpha}\left(\mathbf{r}_{l+1}^{s}, \Theta\right)\right]-\bar{h}_{m}(\Theta)\right]=0, \\
& \lim _{k, n \rightarrow \infty} \frac{1}{k} \sum_{l=n}^{n+k-1}\left[\mathbb{E}_{n}\left[\hat{r}_{m, l+1}^{\alpha} I_{m}^{\alpha}\left(\mathbf{r}_{l+1}^{s}, \Theta\right)\right]-\overline{\hat{h}}_{m}(\Theta)\right]=0
\end{aligned}
$$

in the sense of probability. There are small positive $\delta$ and $\delta_{1}$ such that for every $m \leq M$

$$
\begin{aligned}
& P\left\{\frac{r_{m, k}^{s}}{d_{m}} \geq \frac{r_{j, k}^{s}}{d_{j}-\delta}+\delta_{1}, j \neq m\right\}>0, \quad \text { if } \alpha=1 \\
& P\left\{\frac{r_{m, k}^{s}}{d_{m}^{1-\alpha}} \geq \frac{r_{j, k}^{s}}{\left(d_{j}-\delta\right)^{1-\alpha}}+\delta_{1}, j \neq m\right\}>0, \quad \text { else. }
\end{aligned}
$$

A.3 True, signaled rates $\left\{\left(\mathbf{r}_{l}, \mathbf{r}_{l}^{s}\right) ; l \leq \infty\right\}$ are defined on some compact set and have bounded density.

Remarks VIII-1: The assumption A.1 can be ensured as in Lemma 4 given the assumption A.3.

\section{Appendix B : Proofs !!}

Proof of Lemma 1 : Since $\Gamma^{\alpha}$ is a concave function,

$G^{\alpha}(\beta)-G^{\alpha}\left(\beta^{*}\right) \leq \sum_{m}\left[\theta_{m}(\beta)-\theta_{m}\left(\beta^{*}\right)\right] d \Gamma^{\alpha}\left(\theta_{m}\left(\beta^{*}\right)\right)$. 
From (3) $\beta^{*}$ maximizes the function

$$
\begin{aligned}
\beta & \mapsto \sum_{m} \theta_{m}(\beta) d \Gamma^{\alpha}\left(\theta_{m}\left(\beta^{*}\right)\right) \\
& =\mathbb{E}_{\mathbf{h}}\left[\sum_{m} f\left(h_{m}\right) d \Gamma^{\alpha}\left(\theta_{m}\left(\beta^{*}\right)\right) \beta(m \mid \mathbf{h})\right]
\end{aligned}
$$

over $\mathcal{D}$ and hence we have,

$$
\sum_{m}\left[\theta_{m}(\beta)-\theta_{m}\left(\beta^{*}\right)\right] d \Gamma^{\alpha}\left(\theta_{m}\left(\beta^{*}\right)\right) \leq 0 .
$$

This along with (32) proves that $\beta^{*}$ is a global maximizer of the objective function in (2) over domain $\mathcal{D}$ and hence is a $\alpha$-fair solution (2).

Most of the times there may not be a unique global optimizer for the $\alpha$-fair objective function. However, by uniqueness of $\Theta^{*}$ and by Lemma 3 the rest of the proof follows.

Lemma 3: If there is a BS policy $\beta$ which is inefficient in the following sense: without loss of generality consider the mobile indexed by 1 . If there exists an $h_{1}, h_{1}^{\prime} \in \mathcal{H}_{1}$ and $\overline{\mathbf{h}}_{-1} \in$ $\Pi_{m>1} \mathcal{H}_{m}$ such that

$$
0<\beta\left(1 \mid h_{1}, \overline{\mathbf{h}}_{-1}\right) \leq \beta\left(1 \mid h_{1}^{\prime}, \overline{\mathbf{h}}_{-1}\right)<1 \text { when } f\left(h_{1}\right)>f\left(h_{1}^{\prime}\right)
$$

then one can construct another BS policy $\tilde{\beta}$ which would be better : which yields $\theta_{m}(\tilde{\beta})=\theta_{m}(\beta)$ for all $m>1$ and $\theta_{1}(\tilde{\beta})>\theta_{1}(\beta)$. One can construct a better policy even if there exists an $h_{1}, h_{1}^{\prime} \in \mathcal{H}_{1}$ and $\overline{\mathbf{h}}_{-1} \in \Pi_{m>1} \mathcal{H}_{m}$ such that

$0 \leq \beta\left(1 \mid h_{1}, \overline{\mathbf{h}}_{-1}\right)<\beta\left(1 \mid h_{1}^{\prime}, \overline{\mathbf{h}}_{-1}\right) \leq 1$ when $f\left(h_{1}\right)>f\left(h_{1}^{\prime}\right)$.

Proof : We first construct a better policy for the condition (33). Define a new policy $\tilde{\beta}$ : for all $m$, let

$\tilde{\beta}(m \mid \mathbf{h})=\beta(m \mid \mathbf{h})$ when $\mathbf{h} \neq\left(h_{1}, \overline{\mathbf{h}}_{-1}\right)$ or $\mathbf{h} \neq\left(h_{1}^{\prime}, \overline{\mathbf{h}}_{-1}\right)$.

We will pickup constants $\left\{\epsilon_{m, 1}\right\},\left\{\epsilon_{m, 2}\right\}$ such that for all $m$

$$
\begin{aligned}
& \tilde{\beta}\left(m \mid h_{1}, \overline{\mathbf{h}}_{-1}\right)=\beta\left(m \mid h_{1}, \overline{\mathbf{h}}_{-1}\right)+\epsilon_{m, 1} \quad \text { and } \\
& \tilde{\beta}\left(m \mid h_{1}^{\prime}, \overline{\mathbf{h}}_{-1}\right)=\beta\left(m \mid h_{1}^{\prime}, \overline{\mathbf{h}}_{-1}\right)+\epsilon_{m, 2} .
\end{aligned}
$$

and such that the constructed policy $\tilde{\beta}$ satisfies the requirements of the lemma. First we note that, the sum $\sum_{m} \epsilon_{m, j}$ need to be zero for both $j=1,2$, i.e., $\sum_{m} \epsilon_{m, j}=0$. This is required because the newly constructed policy should satisfy $\sum_{m} \tilde{\beta}(m \mid \mathbf{h})=1$ for all $\mathbf{h} \in \Pi_{m} \mathcal{H}_{m}$. Let $\mathbf{h}_{-1, m}$ represent the component of $\mathbf{h}_{-1}$ corresponding to $m^{\text {th }}$ user. Then since,

$$
\begin{aligned}
& \theta_{m}(\tilde{\beta})= \theta_{m}(\beta)+\epsilon_{m, 1} f\left(\overline{\mathbf{h}}_{-1, m}\right) p_{h_{1}}\left(h_{1}\right) p_{\mathbf{h}_{-1}}\left(\overline{\mathbf{h}}_{-1}\right) \\
&+\epsilon_{m, 2} f\left(\overline{\mathbf{h}}_{-1, m}\right) p_{h_{1}}\left(h_{1}^{\prime}\right) p_{\mathbf{h}_{-1}}\left(\overline{\mathbf{h}}_{-1}\right) \\
&= \theta_{m}(\beta)+\left[\epsilon_{m, 1} p_{h_{1}}\left(h_{1}\right)+\epsilon_{m, 2} p_{h_{1}}\left(h_{1}^{\prime}\right)\right] \\
& f\left(\overline{\mathbf{h}}_{-1, m}\right) p_{\mathbf{h}_{-1}}\left(\overline{\mathbf{h}}_{-1}\right)
\end{aligned}
$$

to make $\theta_{m}(\beta)=\theta(\tilde{\beta})$ we need to set for all $m>1$,

$$
\begin{gathered}
\epsilon_{m, 1}=-\epsilon_{m, 2} \frac{p_{h_{1}}\left(h_{1}^{\prime}\right)}{p_{h_{1}}\left(h_{1}\right)} \text { and hence, } \\
\epsilon_{1,1}=-\sum_{m>1} \epsilon_{m, 1}=-\sum_{m>1} \epsilon_{m, 2} \frac{p_{h_{1}}\left(h_{1}^{\prime}\right)}{p_{h_{1}}\left(h_{1}\right)}=-\epsilon_{1,2} \frac{p_{h_{1}}\left(h_{1}^{\prime}\right)}{p_{h_{1}}\left(h_{1}\right)} .
\end{gathered}
$$

Thus,

$$
\begin{aligned}
\theta_{1}(\tilde{\beta})= & \theta_{1}(\beta)+\epsilon_{1,1} f\left(h_{1}\right) p_{h_{1}}\left(h_{1}\right) p_{\mathbf{h}_{-1}}\left(\overline{\mathbf{h}}_{-1}\right) \\
& +\epsilon_{1,2} f\left(h_{1}^{\prime}\right) p_{h_{1}}\left(h_{1}^{\prime}\right) p_{\mathbf{h}_{-1}}\left(\overline{\mathbf{h}}_{-1}\right) \\
= & \theta_{1}(\beta)+\epsilon_{1,1} p_{\mathbf{h}_{-1}}\left(\overline{\mathbf{h}}_{-1}\right) p_{h_{1}}\left(h_{1}\right)\left[f\left(h_{1}\right)-f\left(h_{1}^{\prime}\right)\right]>0
\end{aligned}
$$

if we set $\epsilon_{1,1}>0$ and because of the following :

- Because $\epsilon_{1,1}>0$, we need $\sum_{m>1} \epsilon_{m, 1}<0$ and thus need at least one $m>1$ such that $\beta\left(m \mid h_{1}, \overline{\mathbf{h}}_{-1}\right)>0$. This is always possible under the hypothesis of the lemma as other wise,

$$
\beta\left(1 \mid h_{1}, \overline{\mathbf{h}}_{-1}\right)=1 \geq \beta\left(1 \mid h_{1}^{\prime}, \overline{\mathbf{h}}_{-1}\right)
$$

and hence contradicts the hypothesis.

- $\epsilon_{1,2}<0$ and hence we need $\beta\left(1 \mid h_{1}^{\prime}, \overline{\mathbf{h}}_{-1}\right)>0$, which is also true because of the hypothesis.

The above two reasons are required to ensure the basic necessary of the policy : $0 \leq \tilde{\beta}(m \mid \mathbf{h}) \leq 1$.

The maximum value of $\epsilon_{1,1}$ is easily seen to be :

$$
\sum_{m>1} \min \left\{\beta\left(m \mid h_{1}, \overline{\mathbf{h}}_{-1}\right), \quad 1-\beta\left(m \mid h_{1}^{\prime}, \overline{\mathbf{h}}_{-1}\right)\right\} .
$$

The last condition can also be taken care in a similar way. If for example, if there exists an $h_{1}, h_{1}^{\prime} \in \mathcal{H}_{1}$ and $\overline{\mathbf{h}}_{-1} \in \Pi_{m>1} \mathcal{H}_{m}$ such that

$0 \leq \beta\left(1 \mid h_{1}, \overline{\mathbf{h}}_{-1}\right)<\beta\left(1 \mid h_{1}^{\prime}, \overline{\mathbf{h}}_{-1}\right)=1$ when $f\left(h_{1}\right)>f\left(h_{1}^{\prime}\right)$, then for all $m>1 \beta\left(m \mid h_{1}^{\prime}, \overline{\mathbf{h}}_{-1}\right)=0$, there exists at least one $\bar{m}>1$ for which $\beta\left(\bar{m} \mid h_{1}, \overline{\mathbf{h}}_{-1}\right)>0$. Then one can chose $\epsilon_{1,1} \frac{p_{h_{1}}\left(h_{1}\right)}{p_{h_{1}}\left(h_{1}^{\prime}\right)}=-\epsilon_{1,2}=\epsilon_{\bar{m}, 2}=-\epsilon_{\bar{m}, 1} \frac{p_{h_{1}}\left(h_{1}\right)}{p_{h_{1}}\left(h_{1}^{\prime}\right)}$ and rest 0 with

$0<\epsilon_{\bar{m}, 2}<\min \left\{\beta\left(\bar{m} \mid h_{1}, \overline{\mathbf{h}}_{-1}\right),\left(1-\beta\left(1 \mid h_{1}, \overline{\mathbf{h}}_{-1}\right)\right) \frac{p_{h_{1}}\left(h_{1}\right)}{p_{h_{1}}\left(h_{1}^{\prime}\right)}\right\}$.

Proof of Lemma 2: As in Lemma 1, for any $\mu$ if there exists a $\beta_{\mu}^{*}$ which satisfies :

$$
\beta_{\mu}^{*}(1 \mid \mathbf{s})=1_{\left\{d \Gamma^{\alpha}\left(\theta_{1}\left(\mu, \beta_{\mu}^{*}\right)\right) f\left(s_{1}\right)>d \Gamma^{\alpha}\left(\theta_{2}\left(\mu, \beta_{\mu}^{*}\right)\right) f\left(s_{2}\right)\right\}}
$$

then it maximizes (12). Define the following for mobile 1 ,

$$
\mu_{1}^{\delta}\left(h_{1, i} \mid h_{1, i^{\prime}}\right)=\left\{\begin{array}{lll}
1_{\left\{i=i^{\prime}\right\}} & \text { if } \quad i^{\prime} \neq i^{*} \\
\delta & \text { if } \quad i=i^{*}-1 \text { and } i^{\prime}=i^{*} \\
1-\delta & \text { if } \quad i^{\prime}=i=i^{*},
\end{array}\right.
$$

where $i^{*}$ is the maximum $i$ satisfying N.2. Define,

$$
\theta_{\text {low }}:=\mathbb{E}_{\mathbf{h}}\left[f\left(h_{1}\right) \beta^{*}(1 \mid m) 1_{\left\{f\left(h_{1}\right) \leq f\left(h_{1, i^{*}}\right\}\right.}\right] .
$$

Mobile 1 can deviate unilaterally from the truth revealing strategy using $\mu_{1}^{\delta}$ and increase its truth revealing utility $\theta_{1}^{\alpha c}$ to a higher utility $U_{1}^{A T A} \geq \theta_{1}^{\alpha c}+\delta f\left(h_{i^{*}}\right)-\theta_{\text {low }}$, whenever the following conditions hold :

$$
\begin{aligned}
\delta f\left(h_{i^{*}}\right) & >\theta_{\text {low }} \\
d \Gamma^{\alpha}\left(\theta_{1}^{\alpha c}\right)-d \Gamma^{\alpha}\left(\theta_{1}^{\alpha c}+\delta f\left(h_{1, i^{*}-1}\right)\right) & <\frac{\eta_{1}}{f\left(h_{1, i^{*}-1}\right)} \\
\text { and } d \Gamma^{\alpha}\left(\theta_{2}^{\alpha c}-\delta u_{2}^{\max }\right)-d \Gamma^{\alpha}\left(\theta_{2}^{\alpha c}\right) & <\eta_{2} \text { with } \\
u_{2}^{\max } & :=\max _{h_{2} \in \mathcal{H}_{2}} f\left(h_{2}\right)
\end{aligned}
$$


with $\eta_{1}+\eta_{2} f\left(h_{2}\right)<\eta$ for all $h_{2} \in \mathcal{H}_{2}$. This is because with the above choice of $\delta$, the mobile 1 , as in cooperative case, will grab the channel with signal $s_{1}=h_{1, i^{*}-1}$ because,

- the corresponding $A S A$ utility

$$
U_{1}^{A S A} \leq \theta_{1}^{\alpha c}+\delta f\left(h_{1, i^{*}-1}\right),
$$

- for every $\alpha$, the function $d \Gamma^{\alpha}($.$) is decreasing in its$ argument and hence

$$
\begin{aligned}
d \Gamma^{\alpha} & \left(U_{1}^{A S A}\right) f\left(h_{1, i^{*}-1}\right) \\
& \geq d \Gamma^{\alpha}\left(\theta_{1}^{\alpha c}+\delta f\left(h_{1, i^{*}-1}\right)\right) f\left(h_{1, i^{*}-1}\right) \\
& \geq d \Gamma^{\alpha}\left(\theta_{1}^{\alpha c}\right) f\left(h_{1, i^{*}-1}\right)-\eta_{1} \\
& \geq d \Gamma^{\alpha}\left(\theta_{2}^{\alpha c}\right) f\left(h_{2}\right)-\eta_{1}+\eta \\
& \geq d \Gamma^{\alpha}\left(\theta_{2}^{\alpha c}-\delta u_{2}^{\max }\right) f\left(h_{2}\right)-\eta_{1}+\eta-\eta_{2} f\left(h_{2}\right) \\
& >d \Gamma^{\alpha}\left(\tilde{\theta}_{2}\left(\beta^{*}\right)\right) f\left(h_{2}\right) \text { for all } h_{2} \in \mathcal{H}_{2},
\end{aligned}
$$

with $\tilde{\theta}_{2}\left(\beta^{*}\right)=U_{2}^{A T A}\left(\mu^{\delta}, \beta_{\mu^{\delta}}^{*}\right)$ representing the new lower utility of the mobile 2 , reduced because of the noncooperation of the mobile 1 , by an amount not more than $\delta u_{2}^{\max }$.

Lemma 4: The function $\bar{h}_{m}(\Theta)$ is continuously differentiable and the function $\overline{\hat{h}}(\Theta)$ is locally Lipschitz, both w.r.t. $\Theta$ for every $m$.

Proof : The result is implied for both the robust policies, if we prove the differentiability of the following functions:

$$
\begin{aligned}
\psi_{m}(\Theta) & :=\mathbb{E}_{\mathbf{s}}\left[\phi_{m}^{\alpha} I_{m}^{\alpha}\left(\Phi^{\alpha}, \Theta\right)\right] \\
\hat{\psi}_{m}(\Theta) & :=\mathbb{E}_{\mathbf{s}}\left[\hat{r}_{m}^{\alpha} I_{m}^{\alpha}\left(\Phi^{\alpha}, \Theta\right)\right]
\end{aligned}
$$

By independence of channel states $\left\{h_{m}\right\}$ across the mobiles,

$$
\begin{aligned}
\psi_{m}(\Theta) & =\mathbb{E}_{\mathbf{s}}\left[\phi_{m}^{\alpha}\left(s_{m}, \theta_{m}\right) I_{m}^{\alpha}\left(\Phi^{\alpha}, \Theta\right)\right] \\
& =\mathbb{E}_{s_{m}}\left[\phi_{m}^{\alpha}\left(s_{m}, \theta_{m}\right) \Pi_{j \neq m} \operatorname{Pr}\left(A_{j}\left(r_{m}^{s}, \Theta\right)\right)\right]
\end{aligned}
$$

where

$$
\begin{aligned}
A_{j}\left(r_{m}^{s}, \Theta\right):=\quad & \left\{r_{j}^{s}: r_{j}^{s} \leq\left(\theta_{j}-\theta_{j}^{\alpha c}\right) \Delta+\right. \\
& \left.\left(r_{m}^{s}-\left(\theta_{m}-\theta_{m}^{\alpha c}\right) \Delta\right)\left(\frac{\theta_{j}+d_{j}}{\theta_{m}+d_{m}}\right)^{\alpha}\right\} .
\end{aligned}
$$

Note in the definition of the sets $A_{j}$, the flag $1_{\left\{\phi_{m}^{\alpha}>0\right\}}$ is dropped, as for the samples with the flag equal to 0 , integrand would any way be zero. The first part of the lemma is proved by BCT if we show that the functions $\left\{\operatorname{Pr}\left(A_{j}\left(s_{m}, \Theta\right)\right)\right\}_{j \neq m}$ and $\phi_{m}^{\alpha}\left(s_{m}, \theta_{m}\right)$ are continuously differentiable (w.r.t. $\Theta$ ) with uniformly bounded derivatives for almost all $s_{m}$. This is immediately evident for $\phi_{m}^{\alpha}$. The same holds for $\left\{\operatorname{Pr}\left(A_{j}\left(s_{m}, \Theta\right)\right)\right\}_{j \neq m}$ by assumption A.3 as,

$$
\begin{aligned}
\frac{\partial \operatorname{Pr}\left(A_{j}\left(r_{m}^{s}, \Theta\right)\right)}{\partial \theta_{l}}= & g_{s_{j}}(\kappa) \frac{d \kappa\left(r_{m}^{s}, \Theta\right)}{d \theta_{l}} \\
\kappa\left(r_{m}^{s}, \Theta\right)= & \left(r_{m}^{s}-\left(\theta_{m}-\theta_{m}^{\alpha c}\right) \Delta\right)\left(\frac{\theta_{j}+d_{j}}{\theta_{m}+d_{m}}\right)^{\alpha} \\
& +\left(\theta_{j}-\theta_{j}^{\alpha c}\right) \Delta
\end{aligned}
$$

for $l=m, j$, where $g_{s_{j}}$ is the (bounded) density of signaled rates $r_{j}^{s}$. Note in the above that the continuous derivative $d \kappa / d \theta_{l}$ will also be uniformly bounded for all $\Theta$ coming from a compact set, because of boundedness of $f$, i.e., of $r_{m}^{s}$.
Easy to see that $\hat{r}_{m}^{\alpha}\left(r_{m}^{s}, \theta_{m}\right)-\hat{r}_{m}^{\alpha}\left(r_{m}^{s}, \theta_{m}^{\prime}\right) \leq \Delta\left|\theta_{m}-\theta_{m}^{\prime}\right|$. Hence, with $C_{f}$ representing the upper bound on function $f$,

$$
\begin{aligned}
& \hat{\psi}^{\alpha}(\Theta)-\hat{\psi}^{\alpha}\left(\Theta^{\prime}\right) \\
&=\mathbb{E}_{\mathbf{s}}\left[\left(\hat{r}_{m}^{\alpha}\left(r_{m}^{s}, \theta_{m}\right)-\hat{r}_{m}^{\alpha}\left(r_{m}^{s}, \theta_{m}^{\prime}\right)\right) I_{m}^{\alpha}\left(\Phi^{\alpha}, \Theta\right)\right] \\
&+\mathbb{E}_{\mathbf{s}}\left[\hat{r}_{m}^{\alpha}\left(r_{m}^{s}, \theta_{m}^{\prime}\right)\left(I_{m}^{\alpha}\left(\Phi^{\alpha}, \Theta\right)-I_{m}^{\alpha}\left(\Phi^{\alpha}, \Theta^{\prime}\right)\right)\right] \\
& \leq \Delta\left|\theta_{i}-\theta_{i}^{\prime}\right| \mathbb{E}_{\mathbf{h}}\left[I_{m}^{\alpha}\left(\Phi^{\alpha}, \Theta\right)\right] \\
&+C_{f} \mathbb{E}_{\mathbf{s}}\left|I_{m}^{\alpha}\left(\Phi^{\alpha}, \Theta\right)-I_{m}^{\alpha}\left(\Phi^{\alpha}, \Theta^{\prime}\right)\right|
\end{aligned}
$$

The lemma follows from the uniform boundedness of the derivative in (34) and the mean value theorem.

\section{APPENDIX C : REMARKS ON CHOICE OF UTILITY :}

Even if a mobile signals more than its true value and the BS attempts to transmit at that higher transmitted rate, the actual rate at which the transmission takes place will still be $f\left(h_{m}\right)$. This is reasonable given the following observations. The reported channel is usually subject to estimation errors and delays, an aspect that we do not consider explicitly in this paper. To address this issue, the BS employs a rate-less code, i.e., starts at an aggressive modulation and coding rate, gets feedback from the mobile after each transmission, and stops as soon as sufficient number of redundant bits are received to meet the decoding requirements. This incremental redundancy technique supported by hybrid ARQ is already implemented in the aforementioned standards (3GPP HSDPA and 1xEV-DO). Then a rate close to the true utility may be achieved. 